\title{
Molecular hydrogen and [Fe II] in Active Galactic Nuclei`
}

\author{
A. Rodríguez-Ardila ${ }^{1}$, M. G. Pastoriza ${ }^{2}$, S. Viegas ${ }^{3}$, T. A. A. Sigut ${ }^{4}$, and A. K. Pradhan ${ }^{5}$ \\ ${ }^{1}$ Laboratório Nacional de Astrofísica, Rua dos Estados Unidos 154, Bairro das Nações, \\ CEP 37500-000, Itajubá, MG, Brazil \\ e-mail: aardila@lna.br \\ 2 Departamento de Astronomia, Instituto de Física, UFRGS, Av. Bento Gonçalves 9500, Porto Alegre, RS, Brazil \\ e-mail: mgp@if.ufrgs.br \\ 3 Instituto de Astronomia, Geofísica e Ciências Atmosféricas, Rua do Matão 1226, CEP 05508-900, São Paulo, SP, Brazil \\ e-mail: viegas@astro.iag.usp.br \\ 4 Department of Physics and Astronomy, The University of Western Ontario, London, ON, N6A 3K7, Canada \\ 5 Department of Astronomy, The Ohio State University, Columbus, OH 43210-1106, USA
}

Received 6 September 2003 / Accepted 13 June 2004

\begin{abstract}
Near-infrared spectroscopy is used to study the kinematics and excitation mechanisms of $\mathrm{H}_{2}$ and [Fe II] lines in a sample of mostly Seyfert 1 galaxies. The spectral coverage allows simultaneous observation of the $J H K$ bands, thus eliminating the aperture and seeing effects that have usually plagued previous works. The $\mathrm{H}_{2}$ lines are unresolved in all objects in which they were detected while the [Fe II] lines have widths implying gas velocities of up to $650 \mathrm{~km} \mathrm{~s}^{-1}$. This suggests that, very likely, the $\mathrm{H}_{2}$ and [Fe II] emission does not originate from the same parcel of gas. Molecular $\mathrm{H}_{2}$ lines were detected in $90 \%$ of the sample, including PG objects, indicating detectable amounts of molecular material even in objects with low levels of circumnuclear starburst activity. Analysis of the observations favors thermal excitation mechanisms for the $\mathrm{H}_{2}$ lines. Indeed, in NGC 3227, Mrk 766, NGC 4051 and NGC 4151, the molecular emission is found to be purely thermal but with heating processes that vary between the objects. Thermal excitation is also confirmed by the rather similar vibrational and rotational temperatures in the objects for which data were available. [Fe II] lines are detected in all of the sample AGN. The [Fe II] $1.254 \mu \mathrm{m} / \mathrm{Pa} \beta \mathrm{ratio}$ is compatible with excitation of the [Fe II] lines by the active nucleus in most Seyfert 1 galaxies, but in Mrk 766 the ratio implies a stellar origin. A correlation between $\mathrm{H}_{2} / \mathrm{Br} \gamma$ and $\left[\mathrm{Fe} \mathrm{II}_{\mathrm{I}} / \mathrm{Pa} \beta\right.$ is found for our sample objects supplemented by data from the literature. The correlation of these line ratios is a useful diagnostic tool in the NIR to separate emitting line objects by their level of nuclear activity. X-ray excitation models are able to explain the observed $\mathrm{H}_{2}$ and part of the [Fe II] emission but fail to explain the observations in Seyfert 2 galaxies. Most likely, a combination of X-ray heating, shocks driven by the radio jet and circumnuclear star formation contributes, in different proportions, to the $\mathrm{H}_{2}$ and [Fe II] lines observed. In most of our sample objects, the [Fe II] $1.257 \mu \mathrm{m} / 1.644 \mu \mathrm{m}$ ratio is found to be $30 \%$ lower than the intrinsic value based on current atomic data. This implies either that the extinction towards the [Fe II]-emitting clouds is very similar in most objects or there are possible inaccuracies in the $A$-values in the Fe II transitions.
\end{abstract}

Key words. galaxies: Seyfert - molecular processes - line: formation - line: profiles - galaxies: active - galaxies: nuclei

\section{Introduction}

One of the fundamental problems in active galactic nuclei (AGN) research is to determine the dominant excitation mechanisms of the narrow line-emitting gas, i.e. whether it is due to non-stellar processes (e.g. photoionization from a central source or shocks from a radio jet) or to stellar processes (e.g. photoionization from OB stars or shocks from supernova remnants). This ambiguity is most evident when interpreting the spectral lines of low-ionization species such as $[\mathrm{Fe}$ II] and

^ Based on observations taken at the Infrared Telescope Facility, which is operated by the University of Hawaii under Cooperative Agreement no. NCC 5-538 with the National Aeronautics and Space Administration, Office of Space Science, Planetary Astronomy Program. molecular hydrogen. Both sets of lines are detected in galaxies displaying varying degrees of nuclear activity, from objects classified as starburst-dominated to those classified as AGN-dominated. Are these lines produced by a unique mechanism in the objects where they are observed, suporting the socalled AGN-starburst connection? or are they formed by different mechanisms (or some combination thereof) in different classes of objects?

Transitions within the ground state of $\mathrm{H}_{2}$ are often bright in near-infrared observations of AGN. The origin of these lines has been highly debated, with no definitive consensus yet reached. Three types of excitation mechanisms may produce the $\mathrm{H}_{2}$ lines: (a) UV fluorescence (e.g. Black \& Van Dishoek 1987); (b) shocks (Hollenbach \& McKee 1989); and (c) X-ray 
illumination (Maloney et al. 1996). UV fluorescence is usually regarded as a non-thermal excitation process and shocks and $\mathrm{X}$-rays as thermal ones. Theoretically, each of these processes produces a different spectrum, and the relative intensities between emission lines of $\mathrm{H}_{2}$ can be used to discriminate the dominant excitation mechanism. So far, however, the results are controversial. For example, Veilleux et al. (1997) claim that shocks associated with nuclear outflows are the most likely source of $\mathrm{H}_{2}$ in AGN while Quillen et al. (1999) relate the $\mathrm{H}_{2}$ emission to local star formation. Very recently, Rigopoulou et al. (2002) carried out a survey of molecular hydrogen emission using a sample of starburst and Seyfert galaxies observed by ISO. They conclude that the $\mathrm{H}_{2}$ emission in Seyferts is most likely a combination of that from photodissociation regions, shock-heated gas and X-ray heated gas, thus favoring thermal excitation over the non-thermal. However, most of the above conclusions have been obtained using a small number of objects with a clear bias towards Seyfert 2. In addition, the aperture used in the ISO observations is usually large which allows the contamination of the nuclear spectra by the host galaxy.

Similar problems, namely production mechanisms and location of the emitting region, are associated with the interpretation of [Fe II] emission in AGN. Forbes \& Ward (1993) found a tight correlation between $[\mathrm{Fe}$ II] and the $6-\mathrm{cm}$ radio emission for both starburst (SB) and Seyfert galaxies. This was taken as strong evidence of shock excitation from the radio jets and/or SNRs for the production of [Fe II] in AGN. However, Simpson et al. (1996) argued that photoionization by the central engine must be the dominant excitation mechanism. More recently, Mouri et al. (2000) supported this scenario by proposing that heating by X-rays is more important in the production of $[\mathrm{Fe} \mathrm{II}]$ and that photoionization by a central source or by shocks can be distinguished by the electron temperature of the [Fe $\mathrm{II}$ ] region: $8000 \mathrm{~K}$ in the former case and $6000 \mathrm{~K}$ for the later. Another useful indicator proposed to distinguish between SB and Seyfert activity for the origin of [Fe II] is the ratio [Fe II] $1.257 \mu \mathrm{m} / \mathrm{Pa} \beta$ (Goodrich et al. 1994; Alonso-Herrero et al. 1997). Observationally, there is an increasing progression in that ratio from pure photoionization to pure shock excitation. In SB galaxies it is observed to be in the range of $0.1-0.5$ (Simpson et al. 1996), in reasonable agreement with the prediction of SB models of Colina (1993), while in AGN it is expected to be in the range of $0.6-3$ if heating by $\mathrm{X}$-rays dominates.

In order to address the above problems and to increase our knowledge of the origin of the $\mathrm{H}_{2}$ and [Fe II] lines, we have observed a sample of 19 Seyfert 1, 2 Seyfert 2 and 1 starburst galaxies. These objects are part of an ongoing program aimed at studying the near-infrared properties of AGN. For most of our target objects, we provide the first reports of molecular and [Fe II] emission. The remaining paper is organized in the following manner: Sect. 2 presents the observations and data reduction; Sect. 3 discusses the kinematics of the molecular and [Fe II] gas inferred from their line profiles; Sect. 4 discusses the origin of the $\mathrm{H}_{2}$ lines; Sect. 5 deals with the emission mechanisms leading to the [Fe II] lines; concluding remarks are given in Sect. 6.

\section{Observations and data reduction}

Near-infrared (NIR) spectra from 0.8-2.4 $\mu \mathrm{m}$ were obtained at the NASA $3 \mathrm{~m}$ Infrared Telescope Facility (IRTF) on April 20-25/2002 with the SpeX spectrometer (Rayner et al. 2003). The detector consisted of a $1024 \times 1024$ ALADDIN 3 InSb array with a spatial scale of $0.15^{\prime \prime} /$ pixel. Simultaneous wavelength coverage was obtained by means of prism cross-dispersers. A $0.8^{\prime \prime} \times 15^{\prime \prime}$ slit was used during the observations, giving a spectral resolution of $360 \mathrm{~km} \mathrm{~s}^{-1}$. For the objects in which the host galaxy is clearly detected on the DSS images, the slit was oriented along the major axis of the target. Otherwise, it was aligned to the parallactic angle in order to minimize slit loses because of differential refraction. During the different nights, the seeing varied between $0.7^{\prime \prime}-1^{\prime \prime}$. Observations were done nodding in an ABBA source pattern with typical individual integration times of $120 \mathrm{~s}$ and total on-source integration times between 30 and $50 \mathrm{~min}$. For some sources, we took spectra on subsequent nights which were, after reduction, combined to form a single spectrum. During the observations, A0 V stars were observed near each target to provide telluric standards at similar air masses. They were also used to flux calibrate the sample. Table 1 shows the log of the observations and the atmospheric conditions during each night. The galaxies are listed in order of right ascension.

The spectral extraction and wavelength calibration procedures were performed using SPEXTOOL, the in-house software developed and provided by the SpeX team for the IRTF community (Cushing et al. 2003; Vacca et al. 2003) ${ }^{1}$. Each pair of $\mathrm{AB}$ observations was reduced individually and the results averaged to provide a final spectrum. A $1.6^{\prime \prime}-2^{\prime \prime}$ aperture, depending on the seeing conditions during the observations, was used to extract the spectra. Observations of an argon arc lamp enabled wavelength calibration of the data; the rms of the dispersion solution was, on average, $0.18 \AA$. No effort was made to extract spectra at positions different from the nuclear region even though some objects show evidence of extended emission. The small beam employed (usually $1.8^{\prime \prime} \times 0.8^{\prime \prime}$ ) and the good seeing during the observations makes this data set truly representative of the nuclear emission, with little contamination of the host galaxy or the extended narrow line region.

Final wavelength-calibrated target spectra were divided by the spectrum of an A0V star observed at a similar airmass to eliminate telluric contamination. Typical airmass differences between the target and the standard were 0.05-0.1. However, despite these small differences, a small residual, mainly in the interval $1.995 \mu \mathrm{m}-2.087 \mu \mathrm{m}$, was found in some objects due to bad telluric band cancellation. After division by the A0V standard, each spectrum was multiplied by a blackbody of temperature equal to the effective temperature of the star to restore the true continuum shape of the target.

Flux calibration was carried out by normalizing to the $K$-band magnitude of the corresponding telluric standard spectrum. We estimate that the uncertainty in this procedure is $\sim 10 \%$ by comparing our calibration with the 2MASS fluxes

\footnotetext{
${ }^{1}$ SPEXTOOL is available from the IRTF web site at http://irtf.ifa.hawaii.edu/Facility/spex/spex.html
} 
Table 1. Observation log and basic galactic properties for the sample. The objects are listed in order of right ascension.

\begin{tabular}{|c|c|c|c|c|c|c|c|c|c|}
\hline $\begin{array}{l}\text { ID } \\
\text { (1) }\end{array}$ & $\begin{array}{l}\text { Galaxy } \\
\text { (2) }\end{array}$ & $\begin{array}{c}\text { Type } \\
\text { (3) }\end{array}$ & $\begin{array}{c}z \\
(4)\end{array}$ & $\begin{array}{c}E(B-V)_{\mathrm{G}} \\
(5)\end{array}$ & $\begin{array}{c}\text { Date of } \\
\text { observation } \\
\text { (6) }\end{array}$ & $\begin{array}{c}\text { On-source } \\
\text { integration } \\
\text { time (s) } \\
\text { (7) }\end{array}$ & $\begin{array}{c}\text { Airmass } \\
\text { (8) }\end{array}$ & $\begin{array}{l}\text { PA } \\
\left({ }^{\circ}\right) \\
(9)\end{array}$ & $\begin{array}{c}r^{a} \\
(\mathrm{pc}) \\
(10)\end{array}$ \\
\hline 1 & Mrk 1210 & Sy2 & 0.01406 & 0.030 & 2002 Apr. 25 & 2700 & 1.25 & 58 & 220 \\
\hline 2 & Mrk 124 & NLS1 & 0.05710 & 0.015 & 2002 Apr. 23 & 2640 & 1.16 & 10 & 990 \\
\hline \multirow[t]{2}{*}{3} & Mrk 1239 & NLS1 & 0.01927 & 0.065 & 2002 Apr. 21 & 1920 & 1.08 & 0.0 & 335 \\
\hline & & & & & 2002 Apr. 23 & 1920 & 1.15 & 0.0 & \\
\hline \multirow[t]{2}{*}{4} & NGC 3227 & Sy 1 & 0.00386 & 0.023 & 2002 Apr. 21 & 720 & 1.00 & 158 & 67 \\
\hline & & & & & 2002 Apr. 25 & 1080 & 1.02 & 158 & \\
\hline 5 & H1143-192 & Sy 1 & 0.03330 & 0.039 & 2002 Apr. 21 & 1920 & 1.31 & 45 & 520 \\
\hline 6 & NGC 3310 & SB & 0.00357 & 0.022 & 2002 Apr. 21 & 840 & 1.21 & 158 & 56 \\
\hline 7 & NGC 4051 & NLS1 & 0.00234 & 0.013 & 2002 Apr. 20 & 1560 & 1.17 & 132 & 37 \\
\hline 8 & NGC 4151 & Sy 1 & 0.00345 & 0.028 & 2002 Apr. 23 & 1800 & 1.10 & 130 & 58 \\
\hline \multirow[t]{2}{*}{9} & Mrk 766 & NLS1 & 0.01330 & 0.020 & 2002 Apr. 21 & 1680 & 1.06 & 112 & 230 \\
\hline & & & & & 2002 Apr. 25 & 1080 & 1.02 & 112 & \\
\hline \multirow[t]{2}{*}{10} & NGC 4748 & NLS1 & 0.01417 & 0.052 & 2002 Apr. 21 & 1680 & 1.29 & 36 & 254 \\
\hline & & & & & 2002 Apr. 25 & 1440 & 1.21 & 36 & \\
\hline 11 & Mrk 279 & NLS1 & 0.03068 & 0.016 & 2002 Apr. 24 & 3600 & 1.54 & 0.0 & 480 \\
\hline 12 & NGC 5548 & Sy1 & 0.01717 & 0.020 & 2002 Apr. 23 & 1920 & 1.05 & 112 & 298 \\
\hline 13 & Mrk 478 & NLS1 & 0.07760 & 0.014 & 2002 Apr. 20 & 3240 & 1.06 & 0.0 & 1200 \\
\hline 14 & NGC 5728 & Sy2 & 0.01003 & 0.101 & 2002 Apr. 21 & 960 & 1.31 & 36 & 160 \\
\hline 15 & PG 1448+273 & QSO & 0.06522 & 0.029 & 2002 Apr. 24 & 2160 & 1.01 & 108 & 1020 \\
\hline 16 & Mrk 291 & NLS1 & 0.03519 & 0.038 & 2002 Apr. 21 & 2520 & 1.04 & 84 & 550 \\
\hline \multirow[t]{2}{*}{17} & Mrk 493 & NLS1 & 0.03183 & 0.025 & 2002 Apr. 20 & 1800 & 1.07 & 0.0 & 500 \\
\hline & & & & & 2002 Apr. 25 & 900 & 1.04 & 0.0 & \\
\hline 18 & PG $1612+261$ & QSO & 0.13096 & 0.054 & 2002 Apr. 23 & 2520 & 1.10 & 107 & 2050 \\
\hline 19 & Mrk 504 & NLS1 & 0.03629 & 0.050 & 2002 Apr. 21 & 2100 & 1.04 & 138 & 570 \\
\hline \multirow[t]{2}{*}{20} & 1H 1934-063 & NLS1 & 0.01059 & 0.293 & 2002 Apr. 21 & 1200 & 1.13 & 150 & 170 \\
\hline & & & & & 2002 Apr. 25 & 720 & 1.17 & 150 & \\
\hline \multirow[t]{3}{*}{21} & Mrk 896 & NLS1 & 0.02678 & 0.045 & 2002 Apr. 23 & 1440 & 1.21 & 150 & 420 \\
\hline & & & & & 2002 Apr. 24 & 1200 & 1.18 & 150 & \\
\hline & & & & & 2002 Apr. 25 & 1200 & 1.17 & 150 & \\
\hline 22 & Ark 564 & NLS1 & 0.02468 & 0.060 & 2000 Oct. 10 & 1500 & 1.05 & 0.0 & 390 \\
\hline
\end{tabular}

${ }^{a}$ Radius of the integrated region.

available in the literature. The agreement in the continuum flux level in the overlap region for the different dispersion orders was excellent with errors less than $1 \%$. The spectra were then corrected for redshift, determined from the average $\langle z\rangle$ measured from the positions of [S III] $0.953 \mu \mathrm{m}, \mathrm{Pa} \delta, \mathrm{He}_{\mathrm{I}} 1.083 \mu \mathrm{m}$, O I $1.128 \mu \mathrm{m}, \mathrm{Pa} \beta$ and $\mathrm{Br} \gamma$. Finally, a Galactic extinction correction as determined from the COBE/IRAS infrared maps of Schlegel et al. (1998) was applied. The value of the Galactic $E(B-V)$ used for each galaxy is listed in Col. 5 of Table 1. Final reduced spectra, in the spectral regions of interest in this work, are plotted in Figs. 1 to 3.

\section{Kinematics of the $\mathrm{H}_{2}$ and [ $\left.\mathrm{Fe} I 1\right]$ lines}

The large spectral coverage and medium spectral resolution of our data $\left(360 \mathrm{~km} \mathrm{~s}^{-1}\right)$ allow us to discuss how the widths of the $\mathrm{H}_{2}$ lines compare to those of other narrow lines. The goal of this analysis is to check if the molecular hydrogen is kinematically linked to the NLR gas, setting important constrains on its location and origin. To our knowledge, no previous results about this issue are available in the literature, mainly because of the lack of adequate spectral resolution in the NIR. Recent NICMOS imaging of $\mathrm{H}_{2}$ emission by Quillen et al. (1999) for a sample of Seyfert galaxies found $\mathrm{H}_{2}$ on scales 

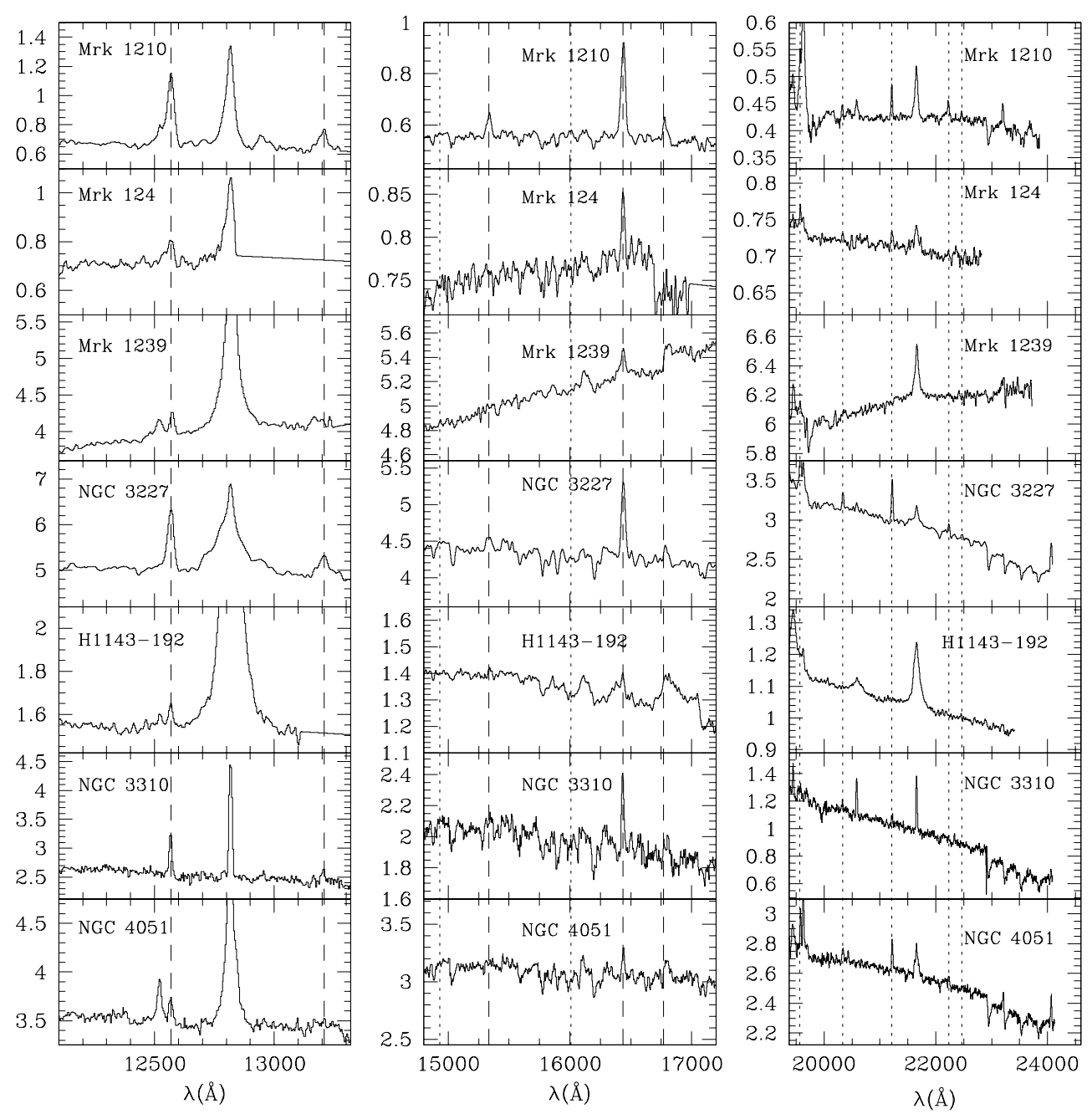

Fig. 1. Final reduced spectra, in the Earth's frame, centred in near $\mathrm{Pa} \beta$ (left panel), the $H$ band (1.60 $\mu \mathrm{m}$, middle panel), and $\mathrm{Br} \gamma$ (right panel). The abscissa is the observed flux in units of $10^{-15} \mathrm{erg} \mathrm{cm}^{-2} \mathrm{~s}^{-1} \AA^{-1}$. The identified [Fe II] (long-dashed lines) and $\mathrm{H}_{2}$ lines (short-dashed lines) are marked in the spectra. Note that in the $H$ band, no $\mathrm{H}_{2}$ lines are detected except in NGC 5728 and NGC 3227 where (1-0)S(7) $1.747 \mu \mathrm{m}$ is visible. We show, however, the expected position of two of the strongest lines predicted by Black \& van Dishoeck's (1987) models.

of a few hundred parsecs from the nucleus for three objects (NGC 5643, NGC 2110 and Mrk 1066). The $\mathrm{H}_{2}$ emission was found to be coincident with [O III] and $\mathrm{H} \alpha+\left[\mathrm{N}_{\mathrm{II}}\right]$, suggesting that the molecular gas may follow the NLR gas. We can test this connection by examining the the line profiles: if the line widths of the molecular gas are similar to those of other narrow emission lines, then, under the assumption that the widths of the NLR lines reflect the large scale motions of the emitting clouds in the gravitational potential of the central mass, similar widths of molecular and forbidden lines suggest that they are co-spatial.

Table 2 lists the FWHM in $\mathrm{km} \mathrm{s}^{-1}$ measured in [S III] $0.9531 \mu \mathrm{m}$, [Si VI] $1.963 \mu \mathrm{m}$, [Fe II] $1.2567 \mu \mathrm{m}$ and $\mathrm{H}_{2}(1,0) \mathrm{S}(1) 2.121 \mu \mathrm{m}$. These values were obtained by fitting a Gaussian to the observed profile and then adopting the $F H W M$ of the fit as representative of the FWHM of the line. The software LINER (Pogge \& Owen 1993) was used for this purpose. Afterwards, we corrected the measurements for instrumental resolution. In all cases, the lines in Table 2 were well represented by a single Gaussian component. For [S III],
[Fe II] and $\mathrm{H}_{2}(1,0) \mathrm{S}(1) 2.121 \mu \mathrm{m}$, no statistically significant shifts relative to the fiducial $z$ were found while the centroid of [ $\mathrm{Si} \mathrm{VI}$ ] was observed to be blueshifted by up to a few hundreds $\mathrm{km} \mathrm{s}^{-1}$ in some objects, as is typical for coronal lines (Penston et al. 1984). Because this latter line is out of the main focus of this paper, we will not make further comments about its shifts. Typical errors, based on the uncertainties in the placement of the continuum, are $\sim 30 \mathrm{~km} \mathrm{~s}^{-1}$. We have chosen $\mathrm{H}_{2}(1,0) \mathrm{S}(1) 2.121 \mu \mathrm{m}$ as representative of molecular hydrogen because it is one of the strongest $\mathrm{H}_{2}$ lines in the NIR. In addition, it is isolated from other emission features, minimizing the effects of blending. Note that the the width and shape of other $\mathrm{H}_{2}$ lines within a given galaxy are rather similar to those of $\mathrm{H}_{2} 2.121 \mu \mathrm{m}$. No $\mathrm{H}_{\mathrm{I}}$ lines were used in this analysis because, for more than half of the sample, the deblending of the observed profile into the components emitted by the NLR and BLR is highly uncertain.

The results show that in all objects the width of $\mathrm{H}_{2}(1,0) \mathrm{S}(1)$ is at the limit of the spectral resolution $\left(360 \mathrm{~km} \mathrm{~s}^{-1}\right)$ while $[\mathrm{Fe} \mathrm{II}]$ is resolved in $50 \%$ of the sample. 

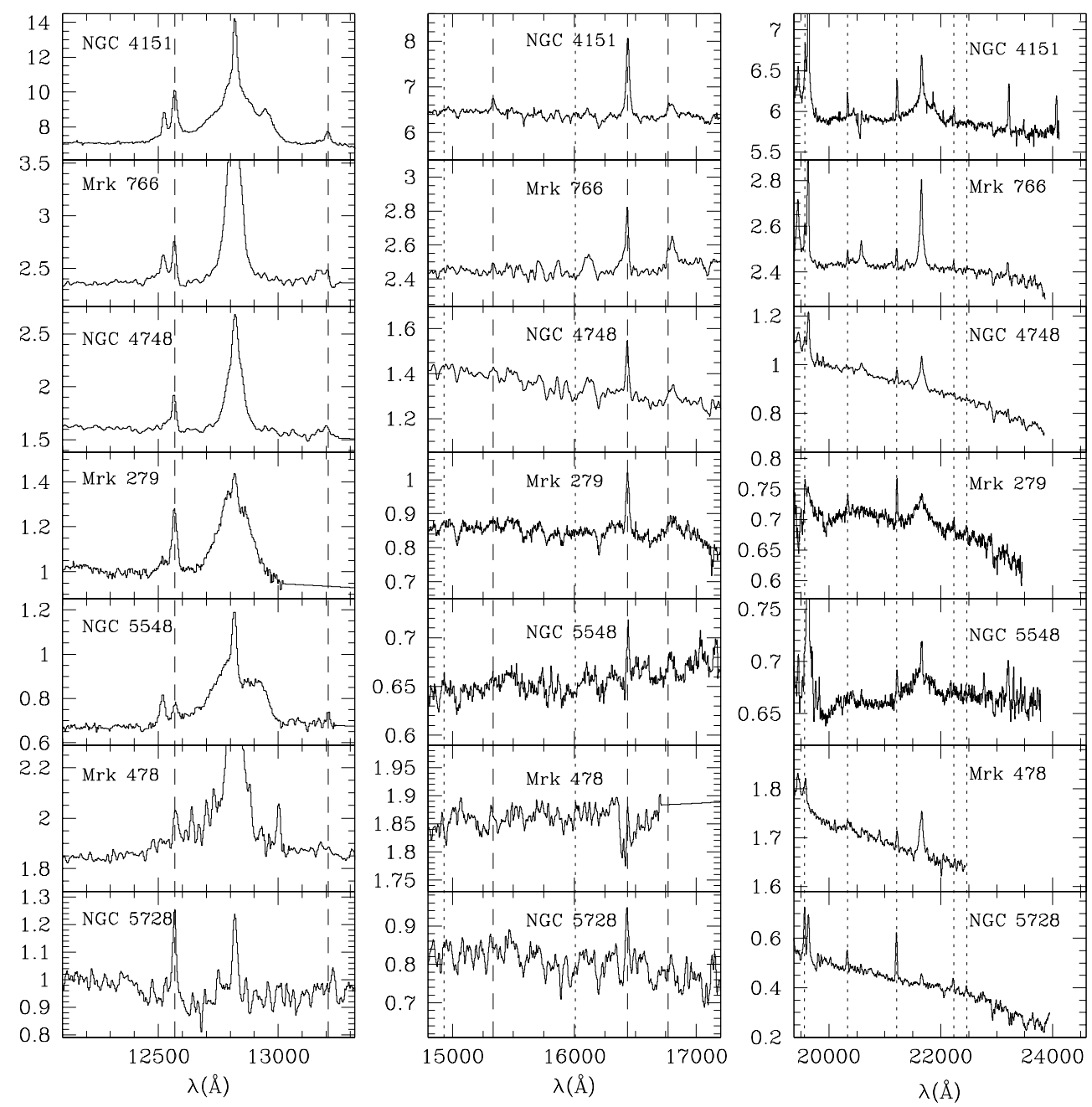

Fig. 2. Same as Fig. 1.

[S III] $0.953 \mu \mathrm{m}(\mathrm{IP}=23.3 \mathrm{eV})$, considered the NIR counterpart of [O III] $5007 \AA$, is unresolved in $60 \%$ of the sample, and in the remaining $40 \%$, the trend is to have similar or larger width than [Fe II]. The coronal line [Si vI] $1.963 \mu \mathrm{m}$ (IP = $167 \mathrm{eV})$, when resolved, is significantly broader than $\mathrm{H}_{2}(1,0) \mathrm{S}(1)$ and, except for a few cases, is also broader than [Fe II]. Overall, in those objects in which the forbidden lines were resolved, we found increasing line widths with increasing ionization potential of the emitting species. A similar trend using optical and NIR lines was found by Rodríguez-Ardila et al. (2002). They showed, for a small sample of Seyfert 1 galaxies, a positive correlation between $F W H M$ and ionization potential. In the optical region, this type of correlation has been firmly established for a large number of AGN (Penston et al. 1984; De Robertis \& Osterbrock 1984; Evans 1988). This trend is usually interpreted as a stratification of the emitting regions in the sense that the most highly ionized gas is located closer to the central source than low-ionization/neutral NLR gas.

NGC 3227, NGC 4151 and PG 1612+261 are counterexamples to the above trend. In these objects, [Fe II] is the broadest NLR line, even when compared to high ionization lines such as [S Ix] $1.252 \mu \mathrm{m}(\mathrm{IP}=328 \mathrm{eV})$. From a kinematical point of view, the bulk of the $[\mathrm{Fe} \mathrm{II}]$ and $\mathrm{H}_{2}$ emission cannot originate in the same volume of gas. Part of [Fe II] must arise from an additional source. We suggest that this extra source may be associated with shock excitation from the radio jet. In fact, Knop et al. (1996) proposed this scenario for NGC 4151. They found [Fe II] broader than the narrow component of $\mathrm{Pa} \beta$ and $[\mathrm{S}$ IX]; the latter two lines are also unresolved in their nuclear spectrum. Knop's $F W H M$ for [Fe II] in NGC 4151 is close to ours (434 $\mathrm{km} \mathrm{s}^{-1}$ vs. $505 \mathrm{~km} \mathrm{~s}^{-1}$, respectively).

The smaller values of the $F W H M$ of $\mathrm{H}_{2}$ relative to other narrow lines may suggest that the molecular gas is concentrated in the external/extended NLR or possibly even in the host galaxy, where the gravitational effects of the black hole on the emitting gas are lower than in the BLR. This scenario seems unlikely, however, because for the large majority of the objects the size of the integrated region covered by the spectra is no larger than $500 \mathrm{pc}$ (see Col. 10 of Table 1). The alternative is to consider that the molecular gas is not kinematically linked to the NLR gas even though the two are co-spatial.

NGC 3227 supports this scenario. Figure 4a shows a comparison of the line profiles of $\mathrm{H}_{2} 2.121 \mu \mathrm{m}$ and [Fe II] $1.257 \mu \mathrm{m}$. It can be seen that the latter line is much broader than the former although both are emitted from a region $\sim 70 \mathrm{pc}$ in radius. Note that [Fe II] $1.257 \mu \mathrm{m}$ is slightly asymmetric towards 

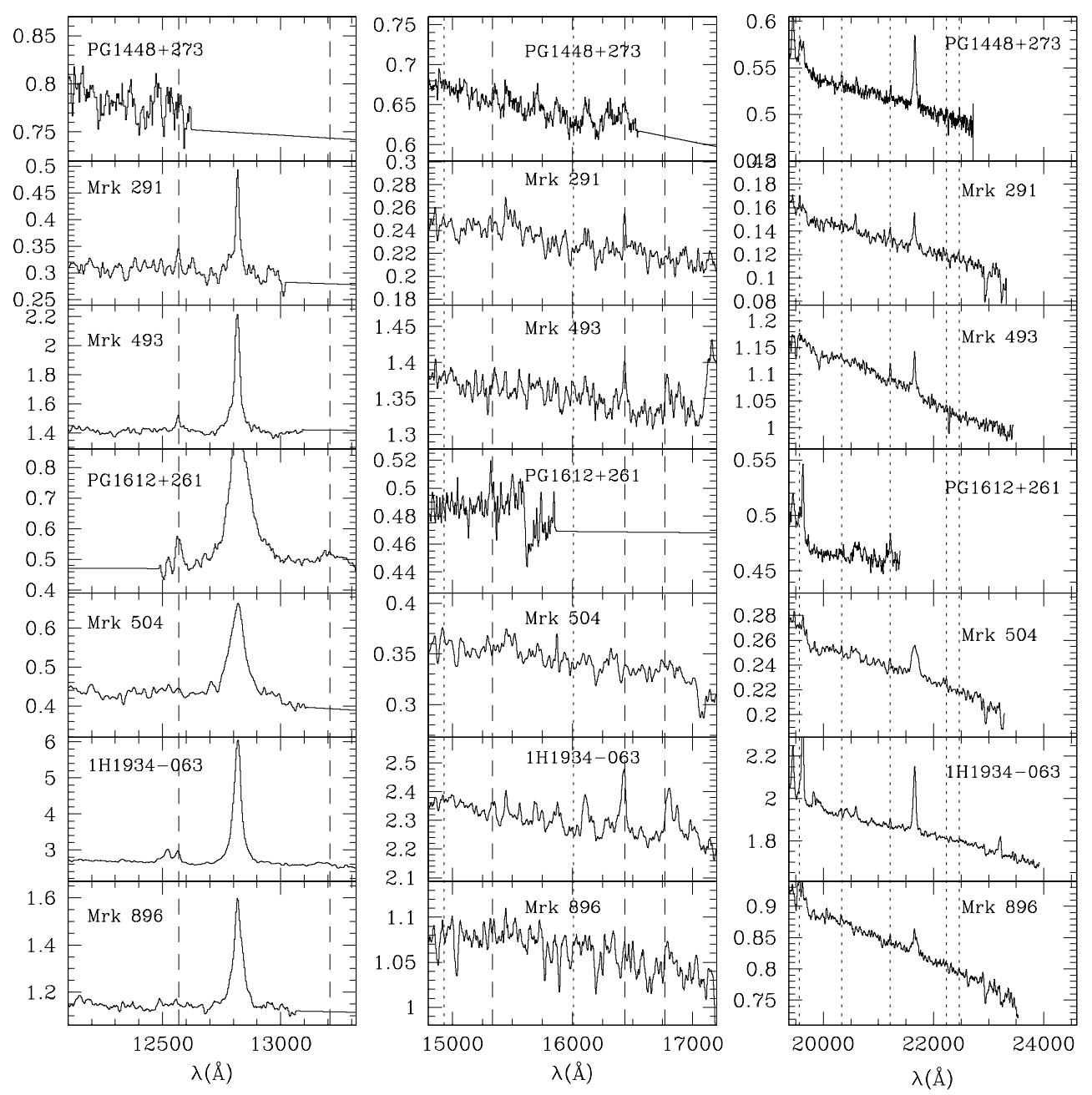

Fig. 3. Same as Fig. 1.

the blue, mainly in the wing of the line, but it is not possible to tell from the data if the asymmetry is related to [Fe $\mathrm{II}]$ or it is due to He I $1.252 \mu \mathrm{m}$. NICMOS imaging in $\mathrm{H}_{2}(1-0) \mathrm{S}(1) 2.121 \mu \mathrm{m}$ carried out by Quillen et al. (1999) on this object shows that the molecular hydrogen emission originates in a $100 \mathrm{pc}$ diameter disk that is not directly associated with either the [O III] emission or the $18 \mathrm{~cm}$ radio emission. Moreover, Rodríguez-Ardila $\&$ Viegas (2003) detected $3.3 \mu \mathrm{m}$ PAH emission in the inner $120 \mathrm{pc}$ of this object, implying circumnuclear star formation within that region probably related to the disk of molecular gas. The differences in line widths reported here support the view that both the NLR and the molecular gas are co-spatial but emitted by different volumes of gas and most probably originated from different excitation mechanisms.

Mrk 1210 is another interesting case for a kinematical decoupling of the molecular gas. The forbidden NLR lines are remarkably similar in FWHM (see Table 2); however, $\mathrm{H}_{2}$ (1-0)S(1) $2.121 \mu \mathrm{m}$ is significantly narrower, almost half the $F W H M$ value of the [Fe II] $1.257 \mu \mathrm{m}$ (Fig. 4b). Radio observations in the $\mathrm{CO}(1 \rightarrow 0) 115 \mathrm{GHz}$ and $\mathrm{CO}(2 \rightarrow 1) 230 \mathrm{GHz}$ carried out on this object (Raluy et al. 1998) show a high level of star forming activity within the inner $5 \mathrm{Kpc}$. Moreover, this Seyfert 2 has a strong $\mathrm{H}_{2} \mathrm{O}$ megamaser of nuclear origin, arising from the innermost parsecs of the host galaxy (Braatz et al. 1994). Because our integrated spectrum covers a region of $\sim 200 \mathrm{pc}$ in radius, it is likely that it includes a significant contribution from the circumnuclear starburst, and the $\mathrm{H}_{2}$ emission we observe can be directly associated to this component.

NGC 5728 also provides additional support for the idea of a disconnection between the $\mathrm{H}_{2}$ emission and the NLR. This AGN is a classic example of a Seyfert 2 galaxy with a biconical emission line region separated by a dark band; the nucleus lies behind, at the point defined by the apexes of the two ionization cones (Wilson et al. 1993). The ionization cones are essentially collinear with the position angle of the brighter SE cone being $118^{\circ}$. In addition to the cones, the nuclear region of NGC 5728 shows other interesting features. In red and green optical continuum images presented by Wilson et al. (1993), there are two miniature bars delineated by four main knots of emission, making the central region of NGC 5728 very complex with several decoupled dynamical components. In its $K$-band spectrum, NGC 5728 shows a remarkable $\mathrm{H}_{2}$ emission; it is the only object in which the $\mathrm{H}_{2}$ emission is stronger than $\mathrm{Br} \gamma$. Our spectrum covers the inner $320 \mathrm{pc}$ with the slit oriented along the larger bar $(\mathrm{PA}=$ $36^{\circ}$ ), almost perpendicular to the ionization cones and along the 
Table 2. $F W H M$ (in $\mathrm{km} \mathrm{s}^{-1}$ ), corrected for instrumental resolution, for $\mathrm{H}_{2}$ and other important lines measured in spectra sample.

\begin{tabular}{|c|c|c|c|c|c|}
\hline Galaxy & $\begin{array}{c}{\left[\mathrm{S}_{\mathrm{III}}\right]} \\
0.9530 \mu \mathrm{m}\end{array}$ & $\begin{array}{c}{[\mathrm{Si} \mathrm{vI}]} \\
1.963 \mu \mathrm{m}\end{array}$ & $\begin{array}{c}{\left[\mathrm{Fe}_{\mathrm{II}}\right]} \\
1.257 \mu \mathrm{m}\end{array}$ & $\begin{array}{c}\mathrm{H}_{2}(1,0) \mathrm{S}(1) \\
2.121 \mu \mathrm{m}\end{array}$ & $\begin{array}{c}\mathrm{O}_{\mathrm{I}} \\
1.128 \mu \mathrm{m}\end{array}$ \\
\hline Mrk 1210 & 685 & 660 & 650 & 380 & $\ldots$ \\
\hline Mrk 124 & 420 & 360 & 420 & 395 & 1330 \\
\hline Mrk 1239 & 865 & $\ldots$ & 456 & $\ldots$ & 1160 \\
\hline NGC 3227 & 470 & 420 & 560 & 360 & 3110 \\
\hline H 1143-192 & 360 & 556 & 404 & $\ldots$ & 1630 \\
\hline NGC 3310 & 360 & $\ldots$ & 360 & 360 & $\ldots$ \\
\hline NGC 4051 & 360 & 360 & 360 & 360 & 880 \\
\hline NGC 4151 & 360 & 360 & 505 & 370 & 3520 \\
\hline Mrk 766 & 360 & 390 & 370 & 370 & 1270 \\
\hline NGC 4748 & 360 & 590 & 440 & 370 & 1550 \\
\hline Mrk 279 & 610 & $\ldots$ & 440 & 370 & 2790 \\
\hline NGC 5548 & 360 & 480 & 435 & 360 & 4860 \\
\hline Mrk 478 & 360 & $\ldots$ & 370 & 380 & 1160 \\
\hline NGC 5728 & 405 & 520 & 360 & 360 & $\ldots$ \\
\hline PG $1448+273$ & 370 & 526 & $\ldots$ & 370 & 728 \\
\hline Mrk 291 & 360 & $\ldots$ & 370 & 370 & $\ldots$ \\
\hline Mrk 493 & 360 & $\ldots$ & 380 & 382 & 610 \\
\hline PG 1612+261 & 360 & 450 & 550 & 370 & 1900 \\
\hline Mrk 504 & 617 & 430 & $\ldots$ & 360 & 1630 \\
\hline 1H 1934-063 & 420 & 650 & 550 & 360 & 850 \\
\hline Mrk 896 & 360 & $\ldots$ & 360 & 360 & 1050 \\
\hline Ark 564 & 440 & 530 & 360 & 370 & 600 \\
\hline
\end{tabular}

dark band. This suggest that the $\mathrm{H}_{2}$ emission is associated with the dark band, which serves as a natural reservoir of molecular gas.

We also note that Mrk 1239 and H 1143-192 are the only Seyfert 1 galaxies of our sample with no $\mathrm{H}_{2}$ lines observed in their spectra. As [Fe II] emission and other NLR features are clearly visible in their spectra (see Fig. 1), these objects provide additional support for a separate spatial origin between the $\mathrm{H}_{2}$ and NLR lines.

Finally from Table 2, the molecular and forbidden lines are all significantly narrower than the BLR lines. The last column of Table 2 shows the $F W H M$ of O I $1.128 \mu \mathrm{m}$, a pure BLR feature emitted in the outer portions of that region. The large difference in line widths between $\mathrm{H}_{2}$ and $\mathrm{O}_{\text {I }}$ makes implausible the emission of $\mathrm{H}_{2}$ close to the BLR, i.e. from the torus of gas and dust around the central engine.

\subsection{NIR kinematics vs. optical kinematics}

It is interesting to see how the widths of NIR lines measured in Sect. 3 for the galaxy sample compare to those found in the optical region. This will give us an insight to how the NLR gas is distributed and most importantly, to check if large amounts of high-velocity gas are obscured by dust extinction

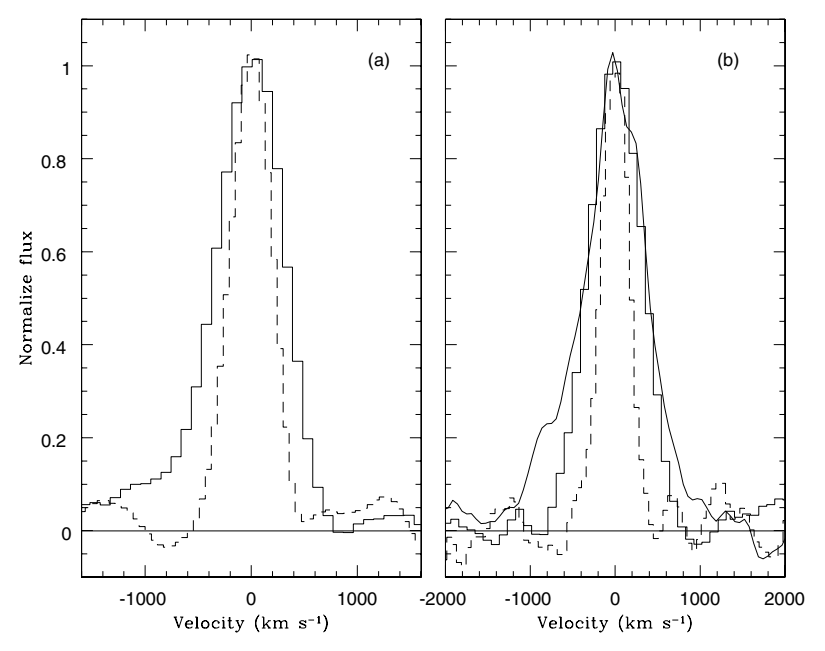

Fig. 4. Comparison of the line profiles of [Fe II] $1.257 \mu \mathrm{m}$ (full histogram) and $\mathrm{H}_{2} 2.122 \mu \mathrm{m}$ (dashed histogram) for a) NGC 3227; and b) Mrk 1210. Note that for this later object, the observed profile of $\operatorname{Br} \gamma$ (solid line) has also been plotted.

at shorter wavelengths. For this analysis we have chosen the lines [O III] $\lambda 5007$ and [S II] $\lambda 6717$. The former can be considered as the optical counterpart of [S III] $\lambda 9531$ while the latter is representative of low-ionization gas such as [Fe II]. Values 
Table 3. Values of $F W H M$, in $\mathrm{km} \mathrm{s}^{-1}$, for optical lines of the galaxy sample taken from the literature.

\begin{tabular}{lcc}
\hline \hline Galaxy & {$[$ O III] $\lambda 5007$} & {$\left[\mathrm{S}_{\text {II }}\right] \lambda 6717$} \\
\hline Mrk 1210 & $494^{2}$ & $276^{2}$ \\
Mrk 1239 & $630^{1}$ & $360^{a, 3}$ \\
NGC 3227 & $460^{5}$ & $427^{5}$ \\
NGC 4051 & $162^{5}$ & $185^{5}$ \\
NGC 4151 & $245^{5}$ & $280^{2}$ \\
Mrk 766 & $180^{5}$ & $159^{5}$ \\
NGC 4748 & $365^{1}$ & $450^{a, 3}$ \\
NGC 5548 & $411^{6}$ & $300^{6}$ \\
Mrk 478 & $920^{1}$ & $\ldots$ \\
PG 1448+273 & $315^{1}$ & $\ldots$ \\
Mrk 493 & $450^{1}$ & $\ldots$ \\
1H 1934-063 & $562^{3}$ & $446^{a, 3}$ \\
Mrk 896 & $315^{1}$ & $\ldots$ \\
Ark 564 & $265^{4}$ & $260^{4}$ \\
\hline
\end{tabular}

${ }^{a}$ FWHM of [N $\left.\mathrm{NI}\right] 26584$.

References - 1. Veron-Cetty et al. (2001); 2. Schulz \& Henkel (2003); 3. Rodríguez-Ardila et al. (2000); 4. Erkens et al. (1997); 5. Veilleux (1991); 6. Moore et al. (1996).

of $F W H M$ for these two lines, extracted from the literature, are listed in Table 3. Note that for most cases, the spectral resolution is higher than that of NIR lines.

Overall, Table 3 supports the conclusions drawn in the previous section. Moreover, no significant broadening of NIR lines relative to the optical ones is detected. The exceptions are Mrk 1210 and Mrk 1239. These two sources are known to have strong polarization due to dust scattering (Tran 1995; Goodrich 1989), supporting the view that high velocity gas is obscured by dust extinction. Note that for Mrk 766 and NGC 4051, the optical lines are half as broad as the NIR ones, but in both cases, the NLR lines are spectroscopically unresolved.

In NGC 3227, NGC 4151, NGC 5548 and 1H 1934-063, the width of [O III] is compatible to that found for [S III], which argues against the presence of high-velocity gas in the visible region, not detected due to extinction. However, $\left[\mathrm{Fe}_{\mathrm{II}}\right]$ is considerably broader than $\left[\mathrm{S}_{\mathrm{II}}\right]$ (or [N $\left.\mathrm{II}\right]$ where the measurements of the sulfur line were not available). This gives additional support to the view that the [Fe $\mathrm{FI}]$ lines are at least partially formed in a region distinct from that of other low-ionization species. Interestingly, the former three galaxies display radiojets, whose interaction with the NLR has been proposed as the source of [Fe II] lines.

\section{Excitation mechanisms of the NIR $\mathrm{H}_{2}$ lines}

The $\mathrm{H}_{2}$ molecule can be excited via three distinct mechanisms: (1) $U V$ fluorescence, where photons with $\lambda>912 \AA$ are absorbed by the $\mathrm{H}_{2}$ molecule and then re-emitted resulting in the population of the various vibration-rotational levels of the ground electronic state (e.g. see Black \& van Dishoek 1987); (2) shocks, where high-velocity gas motions heat, chemically alter, and accelerate the ambient gas resulting in excitation of the $\mathrm{H}_{2}$ molecule (e.g. see Hollenbach \& McKee 1989); and (3) X-ray illumination, where hard Xray photons penetrate deep into molecular clouds, heating large amounts of molecular gas resulting in $\mathrm{H}_{2}$ emission (e.g. see Maloney et al. 1996). Each of these three mechanisms produces a different $\mathrm{H}_{2}$ spectrum; therefore, the relative intensities between emission lines of $\mathrm{H}_{2}$ can help to discriminate the dominant excitation process.

Fluxes of the detected $\mathrm{H}_{2}$ and permitted $\mathrm{H}_{\mathrm{I}}$ lines are listed in Table 4. They were measured by fitting a Gaussian to the observed profile and then integrating the flux under the curve. The LINER software (Pogge \& Owen 1993) was used for this purpose. For a large majority of our sample (16 out of 22 galaxies), these measurements are the first ones reported in the literature. $\mathrm{H}_{2}(1,0) \mathrm{S}(1)$ was detected at a $3 \sigma$ level in 20 out of 22 galaxies, the exceptions being Mrk 1239 and H 1143-182 (see Figs. 1 to 3). It indicates that molecular gas within the inner $500 \mathrm{pc}$ is common in AGN and is not restricted to SB-AGN composite objects. In addition, we clearly detected $\mathrm{H}_{2}(1,0) \mathrm{S}(2)$ for the large majority of AGN. We also detected strong $\mathrm{H}_{2}(1,0) \mathrm{S}(3)$ emission, sometimes larger than $\mathrm{H}_{2}(1,0) \mathrm{S}(1)$, but its flux must be used with caution because it may be severely affected by telluric absorption. Moreover, it is strongly blended with $\operatorname{Br} \delta$ to the blue and [Si vI] $1.963 \mu \mathrm{m}$ to the red. Fluxes for $\mathrm{H}_{2}(1,0) \mathrm{S}(0)$ and $\mathrm{H}_{2}(2,1) \mathrm{S}(1)$ could also be measured at the $3 \sigma$ level in 9 objects; fluxes were derived for the remaining objects using the $3 \sigma$ error as an upper limit. In some Seyfert 1 , it was possible to deblend the contribution of the NLR from the observed $\mathrm{Br} \gamma$ and $\mathrm{Pa} \beta$ lines. Their fluxes appears in Cols. 2 and 3, respectively, of Table 5 .

In order to see how the fluxes listed in Table 4 compare with previous measurements reported in the literature, we have listed in Table 6 fluxes for $\mathrm{H}_{2} 2.121 \mu \mathrm{m}$, [Fe II] $1.64 \mu \mathrm{m}, \mathrm{Br} \gamma$, [Fe II] $1.257 \mu \mathrm{m}$ and $\mathrm{Pa} \beta$ published for a subsample of our objects along with the aperture size used in the observations. If more than one measurement is reported for the same object, they are listed in different lines. This is to avoid mixing data taken under different observing conditions and setups. Clearly, the effect of the extended emission is particularly important for NGC 4151 and NGC 5728. For the remaining galaxies, most of the NIR emission seems to be concentrated in the circumnuclear region. It important to keep in mind that the slit orientation may vary for each entry of Table 6. However, due to the relatively small apertures, its effects on the reported fluxes may not be significant.

In the $H$ band, we detected $\mathrm{H}_{2}(1-0) \mathrm{S}(7) 1.747 \mu \mathrm{m}$ in NGC 5728 and NGC 3227; it was the only $\mathrm{H}_{2}$ line detected in that spectral region. This situation is in contrast with what is observed in planetary nebulae (Rudy et al. 2001) and reflection nebulae (Martini et al. 1997) for example, where bright $\mathrm{H}_{2}$ lines from upper vibrational levels $(v \geq 2)$ are usually present. In these sources, UV fluorescence dominates the production of $\mathrm{H}_{2}$. In fact, the theoretical predictions of Black \& van Dishoeck (1987) based on this mechanism as the main source of energy input show that $\mathrm{H}_{2}$ lines such 
Table 4. Observed fluxes of $\mathrm{H}_{2},[\mathrm{Fe} \mathrm{II}]$ and $\mathrm{H}_{\mathrm{I}}$ lines in units of $10^{-15} \mathrm{erg} \mathrm{cm}^{-2} \mathrm{~s}^{-1}$.

\begin{tabular}{|c|c|c|c|c|c|c|c|c|c|c|}
\hline & {$[\mathrm{Fe}$ II] } & $\mathrm{Pa} \beta^{b}$ & {$[\mathrm{Fe}$ II] } & $\mathrm{H}_{2}$ & $\mathrm{H}_{2}$ & $\mathrm{H}_{2}$ & $\mathrm{Br} \gamma^{b}$ & $\mathrm{H}_{2}$ & $\mathrm{H}_{2}$ & $1.257 \mu \mathrm{m} /$ \\
\hline Galaxy & $1.257 \mu \mathrm{m}$ & $1.282 \mu \mathrm{m}$ & $1.644 \mu \mathrm{m}$ & $1.957 \mu \mathrm{m}$ & $2.0332 \mu \mathrm{m}$ & $2.1213 \mu \mathrm{m}$ & $2.165 \mu \mathrm{m}$ & $2.247 \mu \mathrm{m}$ & $2.223 \mu \mathrm{m}$ & $1.644 \mu \mathrm{m}$ \\
\hline Mrk 1210 & $15.80 \pm 0.70$ & $31.45 \pm 0.88$ & $17.10 \pm 0.64$ & 0 & 0.80 & 2.00 & 6.01 & 0.44 & 0.90 & 0.05 \\
\hline Mrk 124 & $3.16 \pm 0.62$ & $14.6^{a}$ & $2.82 \pm 0.47$ & $0.91 \pm 0.16$ & $0.36 \pm 0.05$ & $0.90 \pm 0.12$ & $2.49 \pm 0.55$ & $<0.18$ & $0.28 \pm 0.08$ & $1.12 \pm 0.29$ \\
\hline Mrk 1239 & $7.90 \pm 0.55$ & $198.6 \pm 2.60$ & $9.72 \pm 1.55$ & $<2.09$ & $<0.93$ & $<0.58$ & $32.0 \pm 1.50$ & $<0.96$ & $<0.82$ & $0.81 \pm 0.14$ \\
\hline NGC 3227 & $41.1 \pm 1.18$ & $185.3 \pm 5.70$ & $41.0 \pm 3.60$ & $20.8 \pm 1.2$ & $7.97 \pm 0.92$ & $17.7 \pm 1.00$ & $30.0 \pm 1.56$ & $2.32 \pm 0.21$ & $3.67 \pm 0.66$ & $1.00 \pm 0.09$ \\
\hline H 1143-192 & $2.54 \pm 0.45$ & $182.0 \pm 2.77$ & $2.45 \pm 0.53$ & $<0.30$ & $<0.23$ & $<0.12$ & $26.7 \pm 1.00$ & $0.24 \pm 0.08$ & $<0.14$ & $1.04 \pm 0.28$ \\
\hline NGC 3310 & $10.70 \pm 0.87$ & $31.74 \pm 1.03$ & $11.12 \pm 1.57$ & $<1.36$ & $1.24 \pm 0.25$ & $1.54 \pm 0.32$ & $10.79 \pm 0.33$ & $<0.32$ & $<0.67$ & 0.15 \\
\hline NGC 4051 & $5.26 \pm 0.81$ & $88.4 \pm 1.80$ & $6.42 \pm 0.97$ & $7.51 \pm 0.62$ & $2.82 \pm 0.70$ & $5.81 \pm 0.37$ & $13.1 \pm 0.84$ & $<0.56$ & $1.52 \pm$ & 0.82 \\
\hline NGC 4151 & $58.8 \pm 2.94$ & $825.6 \pm 11.8$ & $56.2 \pm 1.96$ & $21.2 \pm 2.0$ & $6.84 \pm 0.42$ & $14.7 \pm 0.55$ & $153.8 \pm 7.7$ & $<0.62$ & $5.04 \pm 0.62$ & $1.04 \pm 0.06$ \\
\hline Mrk 766 & $7.69 \pm 0.70$ & $145.0 \pm 1.89$ & $8.20 \pm 0.40$ & $4.39 \pm 0.39$ & $1.73 \pm 0.22$ & $2.36 \pm 0.24$ & $27.4 \pm 0.82$ & $0.30 \pm 0.06$ & $0.81 \pm 0.15$ & $0.94 \pm 0.10$ \\
\hline NGC 4748 & $7.39 \pm 0.37$ & $73.57 \pm 2.84$ & $7.85 \pm 0.61$ & $1.90 \pm 0.42$ & $<0.32$ & $1.59 \pm 0.20$ & $11.32 \pm 0.67$ & $0.40 \pm 0.13$ & $<0.35$ & $0.94 \pm 0.09$ \\
\hline Mrk 279 & $7.22 \pm 0.46$ & $55.2 \pm 2.85$ & $5.36 \pm 0.61$ & $0.67^{a}$ & $1.04 \pm 0.16$ & $1.89 \pm 0.23$ & $11.9 \pm 2.4$ & $0.56 \pm 0.15$ & $0.62 \pm 0.15$ & $1.35 \pm 0.18$ \\
\hline NGC 5548 & 1. & $70.85 \pm 4.45$ & 1.3 & $<1.3$ & $<0$ & 1 & \pm 2.0 & $<0$. & $<0$ & .24 \\
\hline Mrk 478 & $<3.06$ & $82.70^{a}$ & $\ldots$ & $3.73 \pm 0.40$ & $<0.90$ & $1.26 \pm 0.15$ & $6.90 \pm 0.48$ & $\cdots$ & $<0.35$ & $\cdots$ \\
\hline NGC 5728 & $5.08 \pm 0.50$ & $7.37 \pm 1.16$ & $4.96 \pm 0.61$ & $6.04 \pm 0.56$ & $2.40 \pm 0.20$ & $6.28 \pm 0.12$ & $2.11 \pm 0.19$ & $0.89 \pm 0.20$ & $1.68 \pm 0.20$ & $1.02 \pm 0.16$ \\
\hline PG 1448+273 & $\ldots$ & ... & ... & $0.93 \pm 0.07$ & $0.37 \pm 0.08$ & $0.45 \pm 0.08$ & $3.81 \pm 0.17$ & $<0.20$ & $<0.20$ & .. \\
\hline Mrk 291 & $0.91 \pm 0.24$ & $4.27 \pm 0.44$ & $0.80 \pm 0.09$ & $0.30 \pm 0.12$ & $0.22 \pm 0.07$ & $0.47 \pm 0.10$ & $1.68 \pm 0.19$ & $<0.16$ & $<0.10$ & $1.14 \pm 0.33$ \\
\hline Mrk 493 & $1.96 \pm 0.34$ & $3.03 \pm 0.59$ & $1.40 \pm 0.27$ & $\ldots$ & $0.32 \pm 0.07$ & $1.04 \pm 0.20$ & $3.45 \pm 0.25$ & $<0.26$ & $<0.21$ & $1.40 \pm 0.36$ \\
\hline PG 1612+261 & $3.56 \pm 0.25$ & $50.92 \pm 0.98$ & $\ldots$ & $<1.02$ & $<0.07$ & $0.76 \pm 0.17$ & $\ldots$ & $\cdots$ & $\cdots$ & $\ldots$ \\
\hline Mrk 504 & $<0.72$ & $21.8 \pm 1.22$ & $<0.20$ & $<0.32$ & $0.15 \pm 0.04$ & $0.35 \pm 0.06$ & $3.48 \pm 0.45$ & $<0.13$ & $0.20 \pm 0.06$ & ... \\
\hline 1H 1934-063 & $7.24 \pm 0.24$ & $159.0 \pm 1.51$ & $7.46 \pm 1.05$ & $2.38 \pm 0.65$ & $1.80^{a}$ & $1.23 \pm 0.20$ & $18.6 \pm 0.72$ & $0.31 \pm 0.10$ & $<0.10$ & $0.97 \pm 0.14$ \\
\hline Mrk 896 & $<0.40$ & $21.5 \pm 0.69$ & $<0.53$ & $0.41^{a}$ & $0.42 \pm 0.13$ & $0.41 \pm 0.10$ & $3.21 \pm 0.59$ & $0.20 \pm 0.06$ & $<0.19$ & $\ldots$ \\
\hline Ark 564 & $3.87 \pm 0.44$ & $90.9 \pm 1.50$ & $3.90 \pm 0.65$ & $0.80 \pm 0.22$ & $0.53 \pm 0.18$ & $1.24 \pm 0.27$ & $9.74 \pm 0.52$ & $<0.26$ & $<0.22$ & $0.99 \pm 0.20$ \\
\hline
\end{tabular}

${ }^{a}$ Affected by telluric absorption.

${ }^{b}$ Total flux of the line.

Table 5. Fluxes of the narrow component of the permitted $\mathrm{H}_{\mathrm{I}}$ lines and line ratios measured between $\mathrm{H}_{\mathrm{I}}, \mathrm{H}_{2}$ and [Fe II].

\begin{tabular}{lcccc}
\hline \hline Galaxy & $\mathrm{Br} \gamma$ & $\mathrm{Pa} \beta$ & $\mathrm{H}_{2} 2.12 \mu \mathrm{m} / \mathrm{Br} \gamma$ & {$[\mathrm{Fe}$ II] $1.257 \mu \mathrm{m} / \mathrm{Pa} \beta$} \\
\hline Mrk 1210 & $6.01 \pm 0.37$ & $31.45 \pm 0.88$ & $0.33 \pm 0.03$ & $0.50 \pm 0.03$ \\
Mrk 1239 & $8.40 \pm 1.14$ & $77.16 \pm 1.65$ & $<0.07$ & $0.10 \pm 0.01$ \\
NGC 3227 & $6.85 \pm 2.00$ & $23.34 \pm 1.78$ & $2.58 \pm 0.76$ & $1.76 \pm 0.14$ \\
NGC 3310 & $10.79 \pm 0.33$ & $31.74 \pm 1.03$ & $0.14 \pm 0.03$ & $0.33 \pm 0.03$ \\
NGC 4151 & $23.26 \pm 1.49$ & $102.4 \pm 2.52$ & $0.63 \pm 0.05$ & $0.57 \pm 0.03$ \\
Mrk 766 & $7.40 \pm 0.53$ & $27.15 \pm 0.47$ & $0.32 \pm 0.04$ & $0.28 \pm 0.03$ \\
NGC 4748 & $1.39 \pm 0.26$ & $8.77 \pm 0.50$ & $1.14 \pm 0.26$ & $0.84 \pm 0.06$ \\
NGC 5548 & $1.07 \pm 0.16$ & $3.76 \pm 0.45$ & $0.74 \pm 0.15$ & $0.45 \pm 0.09$ \\
NGC 5728 & $2.11 \pm 0.19$ & $7.37 \pm 1.16$ & $2.97 \pm 0.27$ & $0.69 \pm 0.13$ \\
PG 1612+261 & $0.75 \pm 0.15$ & $3.23 \pm 0.18$ & $1.01 \pm 0.30$ & $1.10 \pm 0.10$ \\
\hline
\end{tabular}

as (6-4)Q(1) $1.601 \mu \mathrm{m}$ and (5-3)Q(1) $1.493 \mu \mathrm{m}$ may have fluxes comparable to that of $(1,0) \mathrm{S}(1)$. In our sample, the lack of molecular hydrogen from rotational levels with $v>1$ in the $H$ band suggests that UV fluorescence alone may have little importance in the production of the observed $\mathrm{H}_{2}$ spectrum. It is also possible that these features are diluted by the strong continuum from the AGN but this last hypothesis seems unlikely because [Fe II] $1.64 \mu \mathrm{m}$ is clearly visible in all objects and it often has an intensity comparable to that of $\mathrm{H}_{2}(1,0) \mathrm{S}(1)$.

Studies aimed at investigating the origin of $\mathrm{H}_{2}$ lines carried out on AGN samples show controversial results. Veilleux et al. (1997) claim that shocks associated with nuclear outflows are a likely source of $\mathrm{H}_{2}$, while Quillen et al. (1999) relate the $\mathrm{H}_{2}$ emission to local star formation. Our data can be used to put 
Table 6. Fluxes for the most important NIR lines taken from the literature. In units of $10^{-15} \mathrm{erg} \mathrm{cm}^{-2} \mathrm{~s}^{-1}$.

\begin{tabular}{|c|c|c|c|c|c|}
\hline Galaxy & $\mathrm{H}_{2} 2.121 \mu \mathrm{m}$ & {$\left[\mathrm{Fe}_{\mathrm{II}}\right] 1.64 \mu \mathrm{m}$} & $\mathrm{Br} \gamma$ & {$\left[\mathrm{Fe}_{\mathrm{II}}\right] 1.257 \mu \mathrm{m}$} & $\mathrm{Pa} \beta$ \\
\hline Mrk 1210 & $4.09^{7}$ & $\ldots$ & $7.11^{7}$ & $77.1^{7}$ & $37.4^{7}$ \\
\hline \multirow[t]{4}{*}{ NGC 3227} & $29^{1}$ & $\ldots$ & $\ldots$ & $\ldots$ & $\ldots$ \\
\hline & $\cdots$ & $48 \pm 15^{3}$ & $16.6 \pm 2.6^{3}$ & $\ldots$ & $\cdots$ \\
\hline & $14 \pm 0.7^{6}$ & $32 \pm 1.7^{6}$ & $16 \pm 1.1^{6}$ & $\cdots$ & $\cdots$ \\
\hline & $11.6 \pm 0.08^{8}$ & $20.8 \pm 0.28^{8}$ & $22.1 \pm 0.18^{8}$ & $\ldots$ & $\ldots$ \\
\hline \multirow[t]{2}{*}{ NGC 4051} & $8.7^{1}$ & $\ldots$ & $\ldots$ & $\ldots$ & $\ldots$ \\
\hline & $4.2 \pm 0.5^{6}$ & $2.0 \pm 0.5^{6}$ & $13 \pm 1.9^{6}$ & $\ldots$ & $\ldots$ \\
\hline \multirow[t]{3}{*}{ NGC 4151} & $2.0^{1}$ & $\cdots$ & $\ldots$ & $\ldots$ & $\cdots$ \\
\hline & $\cdots$ & $\ldots$ & $\cdots$ & $36.7 \pm 9^{4}$ & $836.2^{4}$ \\
\hline & $50 \pm 5^{5}$ & $180 \pm 10^{5}$ & $160 \pm 10^{5}$ & $230 \pm 10^{5}$ & $2300 \pm 50^{5}$ \\
\hline Mrk 766 & $2.4^{1}$ & $\ldots$ & $\ldots$ & $\ldots$ & $\ldots$ \\
\hline Mrk 478 & $\ldots$ & $\ldots$ & $15.8 \pm 5^{2}$ & $\ldots$ & $\ldots$ \\
\hline \multirow[t]{2}{*}{ NGC 5728} & $2.6 \pm 0.4^{6}$ & $\ldots$ & $\ldots$ & $\ldots$ & $\ldots$ \\
\hline & $7.01^{7}$ & $\ldots$ & $2.22^{7}$ & $29.9^{7}$ & $21.8^{7}$ \\
\hline
\end{tabular}

References - 1. Ruiz (1997) (1".2 slit width); 2. Rudy et al. (2001). (3" slit width). 3. Schinnerer et al. (2001). (3".6 circular aperture). 4. Knop et al. (1996) $\left(0.75 \times 11^{\prime \prime} 33\right.$ aperture). 5. Thompson (1995) $\left(2^{\prime \prime} \times 10^{\prime \prime}\right.$ aperture). 6. Sossa-Brito et al. (2001) (2" aperture). 7. Veilleux et al. (1997) $\left(3^{\prime \prime} \times 3^{\prime \prime}\right.$ NGC 5728, 6" $\times 3$ Mrk 1210 in J; $3^{\prime \prime} \times 3^{\prime \prime}$ in K). 8. Reunanen et al. (2002) $\left(1^{\prime \prime} \times 1^{\prime \prime} 5\right)$.

stronger constraints on this issue by comparing the observed $\mathrm{H}_{2}$ fluxes with existing models that discriminate between these two possible scenarios. Mouri (1994) proposed that the line ratio $\mathrm{H}_{2}(2,1) \mathrm{S}(1) 2.247 \mu \mathrm{m} /(1,0) \mathrm{S}(1) 2.212 \mu \mathrm{m}$ separates shock-

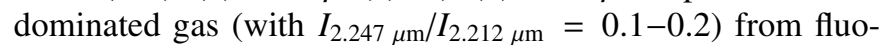
rescent regions (with $I_{2.247} \mu \mathrm{m} / I_{2.212} \mu \mathrm{m} \sim 0.55$ ). However, in dense gas $\left(n \geq 10^{4} \mathrm{~cm}^{-3}\right)$ the collisional de-excitation of the $\mathrm{H}_{2}$ molecule must be taken into account; the spectrum is modified and approaches the thermal spectrum seen in shocks.

Figure 5a shows $(2,1) \mathrm{S}(1) 2.247 \mu \mathrm{m} /(1,0) \mathrm{S}(1) 2.212 \mu \mathrm{m}$ plotted against $(1,0) \mathrm{S}(2) 2.033 \mu \mathrm{m} /(1,0) \mathrm{S}(0) 2.223 \mu \mathrm{m}$. The models of Mouri (1994) are also shown. These lines ratios are reddening insensitive due to the very close wavelengths. A large scatter is observed in both line ratios for the galaxy sample. NGC 3227, NGC 4051, NGC 4151 and Mrk 766 (labelled 4, 7, 8 and 9, respectively) fall near the thermal curve model and indicate excitation temperatures for the thermal component of between $1500 \mathrm{~K}$ and $2500 \mathrm{~K}$. For these galaxies, the $\mathrm{H}_{2}$ emission can be considered purely thermal. For the remaining objects, a mixture of thermal and non-thermal processes are probably at work, with the excitation temperature of the thermal component higher than $1000 \mathrm{~K}$. Interestingly, no $\mathrm{AGN}$ is located in the region occupied by the pure non-thermal UV excitation models, except possibly Mrk 896. However, its error bar makes any statement on this object highly uncertain. As expected, the starburst galaxy NGC 3310 falls far from the locus of points for the non-thermal models. For comparison, data for NGC 253 (Harrison et al. 1998), a well-known starburst galaxy, are also plotted. NGC 253 however, has a thermal excitation temperature below $1000 \mathrm{~K}$, similar only to Mrk 1210 and Mrk 504. Although it is not possible to state what thermal mechanism excites the $\mathrm{H}_{2}$ lines, the results shown in Fig. 5a tell us that the thermal component is dominant over the non-thermal one.

In order to quantify the contribution of the thermal excitation to the $\mathrm{H}_{2}$ lines, we have also plotted in Fig. 5a the predicted line ratios from a mixture of thermal (model S2) and low-density fluorescence models (model 14) taken from Black \& van Dishoek (1987). These two models were empirically mixed in varying proportions and the resultant emission line fluxes were then derived. The calculated line ratios are represented by the dashed line. It connects the models (represented by the triangles) where the percentage of the thermal component decreases in steps of $10 \%$, starting from a model where $90 \%$ is thermal and $10 \%$ UV fluorescence (the first triangle from left to right) up to a contribution of $20 \%$ thermal and $80 \%$ non-thermal. It can be seen that the observations that are scattered across the plot may, in a first approach, be explained by a suitable contribution of thermal and non-thermal processes. Mrk 504 is probably the AGN with the largest contribution of the later mechanism. In decreasing order of importance, we find that UV fluorescence responds for up to $50 \%$ of the observed $\mathrm{H}_{2}$ spectrum in Mrk 279, 30\% in Mrk 1210, 25\% in Mrk 124, and $15 \%$ in NGC 5728.

Figure $5 \mathrm{~b}$ shows $\mathrm{H}_{2}(2-1) \mathrm{S}(1) 2.247 \mu \mathrm{m} /(1,0) \mathrm{S}(1)$ $2.212 \mu \mathrm{m}$ versus $\mathrm{H}_{2}(1-0) \mathrm{S}(3) 1.963 \mu \mathrm{m} /(1-0) \mathrm{S}(1) 2.212 \mu \mathrm{m}$; this is an additional diagnostic diagram proposed by Mouri (1994), equivalent to that of Fig. 5a. However, it has the drawback of including $\mathrm{H}_{2}(1-0) \mathrm{S}(3) 1.957 \mu \mathrm{m}$, which may be strongly affected by telluric absorption bands of $\mathrm{H}_{2} \mathrm{O}$ and $\mathrm{CO}_{2}$ if the redshift of the galaxy is between $z=0.017-0.0398$ or it may be strongly blended with [SivI] $1.963 \mu \mathrm{m}$. In addition, if the broad component of $\operatorname{Br} \delta$ is very conspicuous, it may affect the determination of the continuum level 

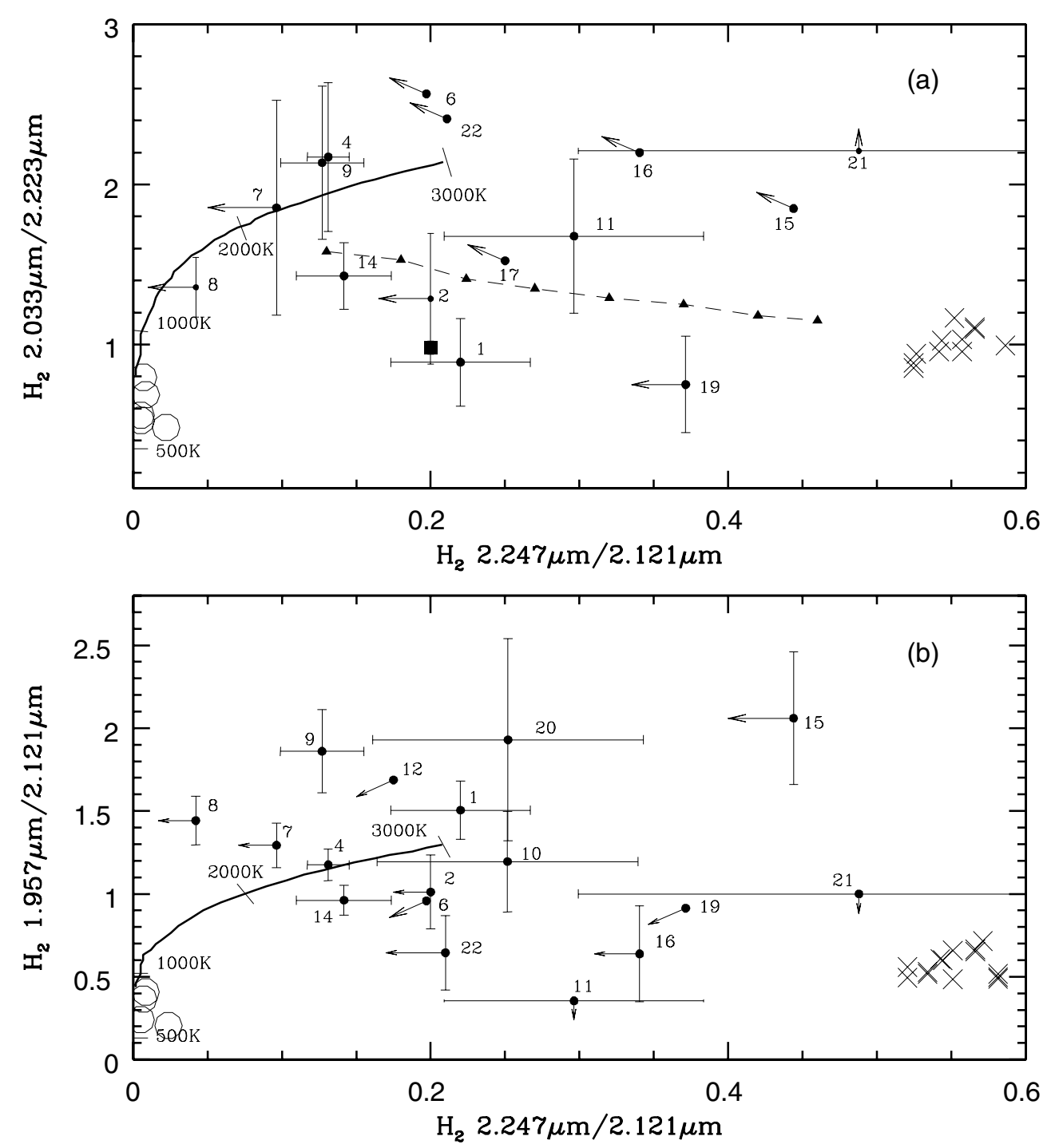

Fig. 5. Plot of a) (2-1)S(1) $2.247 \mu \mathrm{m} /(1-0) \mathrm{S}(1) 2.121 \mu \mathrm{m}$ vs. (1-0)S(2) $2.033 \mu \mathrm{m} /(1-0) \mathrm{S}(0) 2.223 \mu \mathrm{m}$; and b) (2-1)S(1) $2.247 \mu \mathrm{m} /(1-0) \mathrm{S}(1)$ $2.121 \mu \mathrm{m}$ vs. (1-0)S(3) $1.957 \mu \mathrm{m} /(1-0) \mathrm{S}(1) 2.121 \mu \mathrm{m}$. Curves represents thermal emission at 500-3000 K. Crosses are the non-thermal UV excitation models of Black \& Van Dishoek (1987). Open circles are thermal UV excitation models of Sternberg \& Dalgarno (1989). The numbers identify each object according to the notation given in Col. 1 of Table 1 . The black square in a) represents the datum for NGC 253 , a typical starburst galaxy, taken from Harrison et al. (1998). The full triangles, joined by a dashed-line, represents the predicted line ratios from a mixture of thermal and low-density fluorescence models of Black \& Van Dishoek (1987). The percentage of the thermal component decreases in steps of $10 \%$ (and consequently the non-thermal component increases by the same proportion), starting from a model where $90 \%$ is thermal and $10 \%$ UV fluorescence (the first triangle from left to right) up to a contribution of $20 \%$ thermal and $80 \%$ non-thermal (last triangle of the sequence, to the right).

around $\mathrm{H}_{2}(1-0) \mathrm{S}(3) 1.957 \mu \mathrm{m}$. Overall, Fig. 5b reproduces most of the conclusions already drawn: NGC 4051 and NGC 3227 fall on or near the pure thermal excitation curve, and most objects show a thermal excitation temperature in the range $1000-3000 \mathrm{~K}$ indicating a dominance of thermal processes over the non-thermal ones. The departure of Mrk 766 and NGC 4151 from the thermal curve can be explained by the reasons stated above.

\subsection{Rotational and vibrational temperatures}

The main result found in the previous section can be confirmed by deriving rotational and vibrational temperatures for the molecular $\mathrm{H}_{2}$ gas; thermal excitation must give similar rotational and vibrational temperatures, as would be expected for a gas in LTE. Fluorescent excitation, on the other hand, is characterized by a high vibrational temperature and a low rotational temperature; non-local UV photons, not characteristic of the local kinetic temperature, overpopulate the highest energy levels compared to that expected for a Maxwell-Boltzmann population. As a consequence, in photodissociation regions where UV fluorescence dominates, the measured vibrational temperature is $\sim 5000 \mathrm{~K}$ whereas the rotational temperature of the gas is $\sim 10$ per cent of that value (Sternberg \& Dalgarno 1989).

The values of $T_{\text {vib }}$ and $T_{\text {rot }}$ found for the galaxy sample are listed in Table 7 . They were calculated using the fluxes 
Table 7. Vibrational and rotational temperatures found for the molecular hydrogen in the galaxy sample.

\begin{tabular}{|c|c|c|c|c|c|}
\hline Galaxy & $\begin{array}{l}T_{\text {vib }} \\
(\mathrm{K})\end{array}$ & $\begin{array}{l}T_{\text {rot }} \\
(\mathrm{K})\end{array}$ & Galaxy & $\begin{array}{l}T_{\mathrm{vib}} \\
(\mathrm{K})\end{array}$ & $\begin{array}{l}T_{\text {rot }} \\
(\mathrm{K})\end{array}$ \\
\hline Mrk 1210 & $3100 \pm 700$ & $900 \pm 300$ & NGC 5548 & $<2700$ & $\ldots$ \\
\hline Mrk 124 & $<2900$ & $2500 \pm 800$ & NGC 5728 & $2500 \pm 600$ & $1400 \pm 400$ \\
\hline NGC 3227 & $2400 \pm 300$ & $3100 \pm 700$ & PG $1448+273$ & $<5000$ & $<2200$ \\
\hline NGC 3310 & $<3000$ & $<2200$ & Mrk 291 & $<4100$ & $<3300$ \\
\hline NGC 4051 & $<2100$ & $2200 \pm 800$ & Mrk 493 & $<3300$ & $<1600$ \\
\hline NGC 4151 & $<1600$ & $1300 \pm 200$ & Mrk 504 & $<4300$ & $800 \pm 300$ \\
\hline Mrk 766 & $2400 \pm 500$ & $3000 \pm 700$ & 1H 1934-063 & $3300 \pm 1200$ & $\ldots$ \\
\hline NGC 4748 & $3300 \pm 1200$ & $\ldots$ & Mrk 896 & $5500 \pm 2100$ & $<3300$ \\
\hline Mrk 279 & $3700 \pm 1100$ & $1800 \pm 500$ & Ark 564 & $<3000$ & $<4400$ \\
\hline
\end{tabular}

of the observed $\mathrm{H}_{2}$ lines listed in Table 4 and the expressions $T_{\text {vib }} \cong 5600 / \ln \left(1.355 \times I_{1-0 \mathrm{~S}(1)} / I_{2-1 \mathrm{~S}(1)}\right)$ and $T_{\text {rot }} \cong$ $-1113 / \ln \left(0.323 \times I_{1-0 \mathrm{~S}(2)} / I_{1-0 \mathrm{~S}(0)}\right)$ from Reunanen et al. (2002). For Mrk 1239, Mrk 478, H 1143-192 and PG 1612+261, neither of the two temperatures could be derived either because only upper limits to the required fluxes are available or because the lines were not observed.

Table 7 strengths the hypothesis of the $\mathrm{H}_{2}$ lines being purely thermal in NGC 3227, NGC 4051, NGC 4151 and Mrk 766 because $T_{\text {vib }} \simeq T_{\text {rot }}$. In Mrk 1210, Mrk 504, NGC 5728 and Mrk 896, the two temperatures differs appreciably, indicating the presence of non-thermal excitation, as was previously found from the sequence of mixed models of Fig. 5a. The values listed in Table 7 then confirms that for most objects, a mixture of thermal and non-thermal processes contributes to the observed $\mathrm{H}_{2}$ spectrum. Except for Mrk 896 and Mrk 504, it can be stated that thermal processes are the leading excitation mechanism, with a contribution that varies from $60 \%$ up to $100 \%$.

The values for $T_{\text {vib }}$ are also in very good agreement to those derived by Reunanen et al. (2002) for a small sample of AGN in which they found $T_{\text {vib }}$ between $1800 \mathrm{~K}$ and $2700 \mathrm{~K}$. For NGC 3227 , the only object common to both samples, a good agreement is found for $T_{\text {vib }}$ with our results $(2397 \pm 256 \mathrm{~K}$ vs. $1950 \mathrm{~K}$ of Reunanen et al. 2002).

If the main excitation mechanism for $\mathrm{H}_{2}$ in AGN is thermal, it is interesting to examine the possible emission mechanisms as either shocks (produced by supernovae winds or by a radio jet), or UV or X-ray heating are potential candidates for the $\mathrm{H}_{2}$ emission.

Recently, Rodríguez-Ardila \& Viegas (2003) found evidence of starburst activity in Mrk766, NGC 4051, and NGC 3227 by observing the $3.3 \mu \mathrm{m}$ polycyclic aromatic hydrocarbon (PAH) emission in these objects. The beam size covered by their spectra is similar to the one used here. Galaxies with high levels of star formation activity feature strong PAH emission and prominent $\mathrm{H}_{2}$ lines. The relationship between these two characteristics was initially established by Mouri et al. (1990) and later confirmed by Mizutani et al. (1994), who explained it in terms of the physical processes in photodissociation regions associated with relatively massive young stars. Our data provides further support to the hypothesis of the starburst as the origin of the observed molecular hydrogen emission in those three objects. In this scenario, shocks driven by the supernova remnants appears as the most likely source for the $\mathrm{H}_{2}$ excitation. In an AGN with a starburst component, part or all of the observed molecular emission is expected to arise from this process. This seems to be the case of Mrk 766, NGC 4051, and NGC 3227. The predicted $\mathrm{H}_{2} 2-1 \mathrm{~S}(0) / 1-0 \mathrm{~S}(1)$ ratio for the pure shock model (S2) of Black \& van Dishoek (1987) at $T=2000 \mathrm{~K}$ is 0.082 , in marginal agreement to the measured ratios of $0.13 \pm 0.03,0.096$, and $0.13 \pm 0.01$, respectively. Their $\mathrm{S} 2$ model also predicts a $\mathrm{H}_{2} 1-0 \mathrm{~S}(0) / 1-0 \mathrm{~S}(1)$ ratio of 0.21 , well in accord to the values of $0.34 \pm 0.07,0.26 \pm 0.07$, and $0.21 \pm 0.04$, respectively.

Although supernova-driven shocks offer a plausible scenario to explain the pure thermal $\mathrm{H}_{2}$ spectrum observed in those three objects, we cannot ignore that AGN are powerful sources of X-ray emission. It is then expected that X-ray heating may have a non-negligible contribution to the observed $\mathrm{H}_{2}$ flux. Moreover, supernova remnants themselves emits high-energy radiation capable of heating the gas.

For NGC 4151, the non-detection of the $3.3 \mu \mathrm{m}$ feature within the inner 3 ". $8 \times 3$ ". 8 region (Imanishi et al. 1998) makes unlikely the hypothesis of a starburst as the main contributor of the $\mathrm{H}_{2}$ lines. Further efforts to detect signatures of starburst activity in that Seyfert have failed (e.g. Sturm et al. 1999). The analysis of the molecular line profiles carried out in Sect. 3 showed that it is unlikely that $\mathrm{H}_{2}$ is produced by shocks from the radio jet due to the small value of $F W H M$ measured. Thus $\mathrm{X}$-ray heating is the most plausible mechanism for $\mathrm{H}_{2}$ excitation in this object; specific models to test this possibility are discussed in Sect. 5.2.

Finally, we comment on Mrk 1210. For this galaxy, we derived a $T_{\text {rot }} \sim 900 \mathrm{~K}$. We already know that UV fluorescence has, at most, a $30 \%$ contribution. This means that the low $T_{\text {rot }}$ can be attributed to UV heating and this process can be the main source of $\mathrm{H}_{2}$ excitation. UV heating models (Black \& van Dishoek 1987), where FUV photons emitted by OB stars (photodissociation regions) heat the gas, predict excitation temperatures of $\leq 1000 \mathrm{~K}$ for the $\mathrm{H}_{2}$ thermal component. Such low values are typically found in starburst galaxies (Mouri 1994), 
suggesting that the $\mathrm{H}_{2}$ emission observed in Mrk 1210 may primarily come from a circumnuclear starburst. In fact, the position of Mrk 1210 in Fig. 5a is almost coincident with that of NGC 253, a well-known starburst galaxy. Additional evidence giving support to this hypothesis was found in Sect. 3, where the kinematics of the $\mathrm{H}_{2}$ gas pointed out that it was unrelated to the AGN. Further arguments supporting this view come from Storchi-Bergmann et al. (1998) and Schulz \& Henkel (2003) who reported the detection of a Wolf-Rayet (WR) feature around $\lambda 4686 \AA$ in the inner $2^{\prime \prime}$ of Mrk 1210. This aperture coincides with the beam size of our observation. Moreover, WR-features are taken as unambiguous signs for the presence of a starburst (Schulz \& Henkel 2003) and due to the close relationship between $\mathrm{H}_{2}$ and starburst activity (Mouri 1990; Mizutani et al. 1994) it is natural to attribute the $\mathrm{H}_{2}$ emission to this last component. Observations at higher spatial resolution can test this scenario.

In summary, the observational evidence presented up to now shows that the molecular hydrogen lines in AGN are mostly thermal, with a contribution of non-thermal processes typically varying from $15 \%$ to $30 \%$. There is not unique process associated with the thermal component. $\mathrm{H}_{2}$ gas heated by UV photons emitted from photodissociation regions, X-rays from the AGN, and shocks from supernovae remnants arise as the most likely sources of $\mathrm{H}_{2}$ excitation. Our results agree with those of Rigopoulou et al. (2002), found using mid-infrared $\mathrm{H}_{2}$ lines from ISO observations for a sample of Seyfert 2 galaxies.

\section{The origin of the $[\mathrm{Fe} I I]$ lines}

The origin of [Fe II] in active galaxies is highly controversial. It can be produced by shock excitation from the radio jets and/or photoionization from the central source. In addition, fast shocks associated with supernova remnants can be an additional source of [Fe II], as is fairly well established in starburst galaxies (Simpson et al. 1996; Moorwood \& Oliva 1988). As found by Forbes \& Ward (1993), the strength of the [Fe II] emission in the central regions of AGN is tightly correlated with the $6-\mathrm{cm}$ radio emission, and that the same correlation is obeyed for both starburst and Seyfert galaxies. This was taken as evidence that the most likely mechanism for the production of [Fe II] emission in Seyfert galaxies is shock excitation from the radio jets and/or SNRs.

From Table 4 and Figs. 1 to 3 , it can be seen that [Fe II $1.257 \mu \mathrm{m}$ and [Fe II] $1.64 \mu \mathrm{m}$ were detected in all objects of our sample but PG $1448+273$. In the latter AGN, both lines are located in regions of very poor atmospheric transmission, hampering any effort to detect them.

The flux ratios [Fe II] $1.644 \mu \mathrm{m} / \mathrm{Br} \gamma$, or equivalently, [Fe II] $1.257 \mu \mathrm{m} / \mathrm{Pa} \beta^{2}$ can be used to study the origin of the

\footnotetext{
2 The flux ratios [Fe II] $1.644 \mu \mathrm{m} / \mathrm{Br} \gamma$ and [Fe II] $1.257 \mu \mathrm{m} / \mathrm{Pa} \beta$ are equivalent because the two $[\mathrm{Fe}$ II] lines share the same upper level. They are related by the equation [Fe II] $1.257 \mu \mathrm{m} / \mathrm{Pa} \beta=$ $0.231 *[\mathrm{Fe}$ II $] \quad 1.644 \mu \mathrm{m} / \mathrm{Br} \gamma$. The advantage of using [Fe II] $1.257 \mu \mathrm{m} / \mathrm{Pa} \beta$ instead of $\left[\mathrm{Fe}{ }_{\mathrm{II}}\right] 1.644 \mu \mathrm{m} / \mathrm{Br} \gamma$ is that the former is reddening insensitive due to the proximity in wavelength of both lines, serving as a more reliable diagnostic.
}

[Fe II] emission because there is an increasing progression in the ratio from pure photoionization (as in $\mathrm{H}_{\text {II }}$ regions) to pure shock excitation (i.e. supernovae remnants), with starburst and active galaxies located at intermediate values (Mouri et al. 1993; Goodrich et al. 1994; Alonso-Herrero et al. 1997). In starburst galaxies the ratio [Fe $\mathrm{II}] 1.644 \mu \mathrm{m} / \mathrm{Br} \gamma$ is observed to be in the range 0.5-2 (Simpson et al. 1996), in reasonable agreement with the starburst models of Colina (1993) which predicts ratios between 0.1 and 1.4. In these objects, the [Fe II] emission originates in supernova-driven shocks (Mouri et al. 2000). AGN usually show larger values of [ $\mathrm{Fe}$ II $] / \mathrm{Br} \gamma$ than starburst galaxies, probably reflecting the effect of the Seyfert nuclei (Kawara et al. 1988). In fact, X-ray emission, which is dominant in Seyferts, can penetrate deeply into atomic gas and create extended partly ionized regions where [Fe II] can be formed. Models presented by Alonso-Herrero et al. (1997) show that X-rays are able to explain [Fe II]/Br $\gamma$ ratios up to $\sim 20$.

Column 5 of Table 5 lists the flux ratio [Fe II] $1.257 \mu \mathrm{m} / \mathrm{Pa} \beta$ as derived for our galaxy sample. Only the objects in which it was possible to carry out a clean deblending of the narrow component of the permitted lines are included in this analysis. Overall, our line ratios agree with the values found for other samples of Seyfert galaxies, $0.5<$ [Fe II $] 1.257 \mu \mathrm{m} / \mathrm{Pa} \beta<$ 4.6 (see Fig. 3 of Alonso-Herrero et al. 1997). Interestingly, Mrk 766, a NLS1 with a circumnuclear starburst, has a [Fe II] $1.257 \mu \mathrm{m} / \mathrm{Pa} \beta$ ratio similar to that observed in starburst galaxies, indicating that most of $[\mathrm{Fe} \mathrm{II}]$ we detect is of stellar origin. On the other hand, NGC 3227, a classical Seyfert 1 also harboring a circumnuclear starburst, has a high [Fe II] $1.257 \mu \mathrm{m} / \mathrm{Pa} \beta$ ratio, pointing towards an AGN origin. NGC 4151 and NGC 5548 are in the borderline of the starburstAGN division but any contribution from star forming regions would be negligible because of the lack of circumnuclear starburst in these objects.

Although the [Fe II] $1.257 \mu \mathrm{m} / \mathrm{Pa} \beta$ is helpful to discriminate between a stellar and non-stellar origin for [Fe II], it can say little about the excitation mechanisms that can lead to the $\left[\mathrm{Fe}_{\mathrm{II}}\right]$ lines in the latter case. Heating either by X-rays or shocks created by mass outflows from the nuclei (i.e., jets and/or winds interactions with ambient clouds) are plausible scenarios. As proposed by Mouri et al. (2000), these two processes can be discriminated by the electron temperature of the [Fe II] region: $8000 \mathrm{~K}$ in heating by X-rays, and $6000 \mathrm{~K}$ in heating by shocks. Determining the [Fe II] temperature, however, is a difficult task, mostly because some of the diagnostic lines are intrinsically weak and heavily blended with broad adjacent features. The alternative is to examine if X-rays can explain the observed emission. This issue is addressed in detail in Sect. 5.2.

\subsection{Are [Fe $I]$ and $\mathrm{H}_{2}$ related?}

The observational evidence presented throughout this work indicates that $\left[\mathrm{Fe}_{\mathrm{II}}\right]$ and $\mathrm{H}_{2}$ are common features in the nuclear spectra of AGN, although probably originating in different parcels of gas. However, this does not exclude the possibility that both sets of lines originate from a single, dominant mechanism. Moreover, if dust is intermixed with the 
Table 8. Line ratios $\mathrm{H}_{2} 2.121 \mu \mathrm{m} / \mathrm{Br} \gamma$ and [Fe II] $1.257 \mu \mathrm{m} / \mathrm{Pa} \beta$, from AGN taken from the literature.

\begin{tabular}{lccc}
\hline \hline Galaxy & $\mathrm{H}_{2} 2.121 \mu \mathrm{m} / \mathrm{Br} \gamma$ & {$\left[\mathrm{Fe}_{\text {II }}\right] 1.257 \mu \mathrm{m} / \mathrm{Pa} \beta$} & Reference \\
\hline NGC 1386 & $1.14 \pm 0.10$ & $1.59 \pm 0.14$ & 1 \\
NGC 4945 & $3.13 \pm 0.06$ & $0.86 \pm 0.03$ & 1 \\
NGC 5128 & $2.04 \pm 0.10$ & $3.42 \pm 0.16$ & 1 \\
NGC 1097 & $>4.40$ & $>0.43$ & 1 \\
Mrk 1066 & $0.89 \pm 0.06$ & $0.75 \pm 0.04$ & 2 \\
NGC 2110 & $3.17 \pm 0.63$ & $8.11 \pm 0.78$ & 2 \\
NGC 4388 & $0.88 \pm 0.08$ & $0.40 \pm 0.03$ & 2 \\
Mrk 3 & $0.31 \pm 0.04$ & $1.24 \pm 0.04$ & 2 \\
\hline
\end{tabular}

References: (1) Reunanen et al. (2002); (2) Knop et al. (2001).

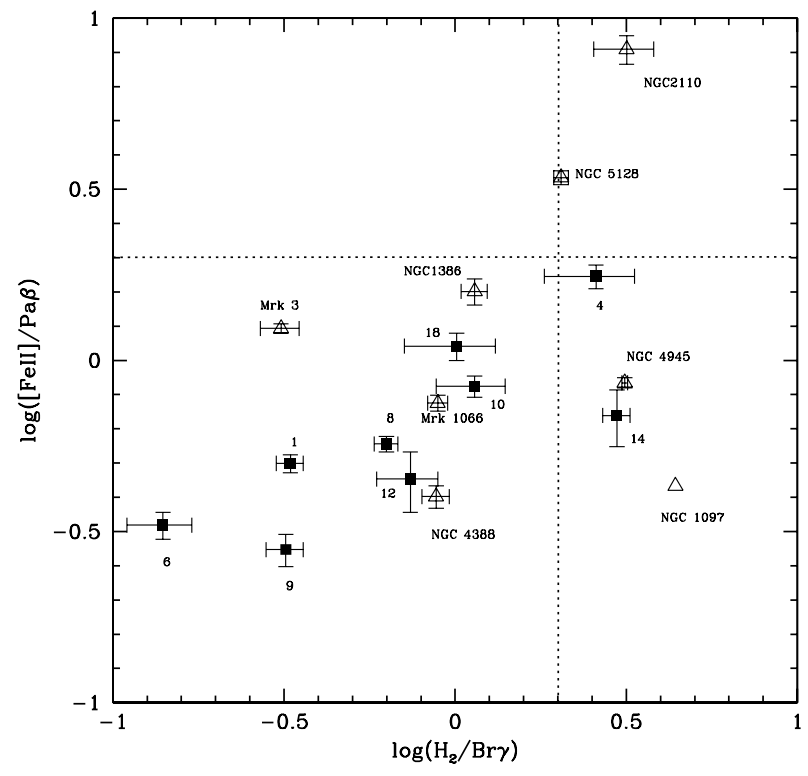

Fig. 6. The ratios $\mathrm{H}_{2} 2.121 \mu \mathrm{m} / \mathrm{Br} \gamma$ and [Fe II] $1.257 \mu \mathrm{m} / \mathrm{Pa} \beta$. Only the Seyfert 1s and NLS1 in which a reliable deblending of the narrow component of the $\mathrm{H}_{\mathrm{I}}$ lines was carried out were plotted. Filled squares represent our data and open triangles data taken from the literature (See Table 8 and text for further details). The number along each data point identifies our galaxies according to the notation given in Col. 1 of Table 1.

line emitting clouds, and there are strong velocity gradients, [Fe II] and $\mathrm{H}_{2}$ lines can appear to have different velocity fields (Knop et al. 2001) even though they are produced in adjacent regions.

In order to examine a possible relationship between [Fe II] and $\mathrm{H}_{2}$, we have plotted in Fig. $6 \mathrm{H}_{2} 2.121 \mu \mathrm{m} / \mathrm{Br} \gamma$ versus [Fe II] $1.257 \mu \mathrm{m} / \mathrm{Pa} \beta$ for our sample along with data taken from the literature for Seyfert 2 galaxies observed with a similar beam size and resolution (see Table 8). Note that the line ratios are completely reddening-free due to their close proximity in wavelength for the lines involved.

Interesting results are seen in Fig. 6. For most objects with both $\mathrm{H}_{2} / \mathrm{Br} \gamma$ and $[\mathrm{Fe} I \mathrm{II}] / \mathrm{Pa} \beta<2$, the two ratios shows a possible, positive correlation. However, for values of either ratio larger than 2, the correlation breaks down and no trend is observed. In addition, no Seyfert 1 galaxy displays extreme values of any of the line ratios plotted in Fig. 6; Both $\mathrm{H}_{2} / \mathrm{Br} \gamma$ and $[\mathrm{Fe} \mathrm{II}] / \mathrm{Pa} \beta>2$ occurs predominantly in Seyfert 2 galaxies.

The correlation observed in Fig. 6 is consistent with the one found by Larkin et al. (1998) in a study of LINERs and other emission line objects, including a few Seyferts. They report a strong linear correlation in the log-log space between $[\mathrm{Fe} \mathrm{II}] / \mathrm{Pa} \beta$ and $\mathrm{H}_{2} / \mathrm{Br} \gamma$, with LINERs displaying the largest ratios and starbust galaxies the smallest ones. Larkin et al. (1998) suggested that $\left[\mathrm{Fe} \mathrm{II}^{\mathrm{I}}\right] / \mathrm{Pa} \beta \sim 1$ and $\mathrm{H}_{2} / \mathrm{Br} \gamma \sim$ 3 mark the end of Seyfert-like nuclei and the beginning of LINER-like objects. The larger sample of Seyfert galaxies employed here allows us to propose that the borderline between Seyfert and LINER activity occurs at $[\mathrm{Fe} \mathrm{II}] / \mathrm{Pa} \beta \sim 2$ and $\mathrm{H}_{2} / \mathrm{Br} \gamma \sim 2$. In this sense, Fig. 6 would serve as a diagnostic diagram in the NIR to separate galaxies according to their level of nuclear activity: starburts occupying the bottom left corner of the plot, with $\left[\mathrm{Fe} \mathrm{II}_{\mathrm{II}}\right] \mathrm{Pa} \beta$ and $\mathrm{H}_{2} / \mathrm{Br} \gamma<0.3$, Seyferts occupying the locus of points with values of either ratio between $0.4-2$, and LINERS showing ratios larger than 2 . We note that, as in our plot, Larkin et al. (1998) found that some of the Seyfert 2 galaxies lie significantly below or above the correlation.

Assuming that $[\mathrm{Fe} \mathrm{II}] / \mathrm{Pa} \beta$ and $\mathrm{H}_{2} / \mathrm{Br} \gamma$ are in fact correlated, interpreting the origin of that correlation is non-trivial. This is mainly because Fig. 6 does not discriminate between the different processes that may give rise to either [Fe II] or $\mathrm{H}_{2}$ in each object. The most straightforward interpretation is that both sets of lines are excited by the same mechanism. It is still possible that more than one mechanism drives the production of [ $\mathrm{Fe}$ II] and $\mathrm{H}_{2}$ within the same object, but that environmental effects dilute the effects of one mechanism over the other (i.e., the presence of a circumnuclear starburst in an AGN).

Recalling that the objects plotted in Fig. 6 are all AGNs, it is natural to consider that the dominant excitation mechanism discussed above is related to the central engine. In particular, X-rays from the nucleus can heat the NLR gas through the large partially ionized zone it creates, driving the [Fe II] and $\mathrm{H}_{2}$ emission. Since $98 \%$ of the iron is tied up in dust grains, this process must free iron through dust destruction and yet not destroy the $\mathrm{H}_{2}$ molecules. Blietz et al. (1994) 
Table 9. Predicted $\mathrm{H}_{2} 2.12 \mu \mathrm{m}$ fluxes from the X-ray models of Maloney et al. (1996) for a density of $10^{5} \mathrm{~cm}^{-3}$.

\begin{tabular}{lcccccc}
\hline \hline & $\log L_{\mathrm{x}}^{1}$ & $N_{\mathrm{Hx}}$ & $N_{\mathrm{H}, \mathrm{NLR}}$ & & \multicolumn{2}{c}{ Predicted $\mathrm{H}_{2} 2.12 \mu \mathrm{m}$} \\
\cline { 6 - 7 } Galaxy & $\left(\mathrm{erg} \mathrm{s}^{-1}\right)$ & $\left(10^{21} \mathrm{~cm}^{-2}\right)$ & $\left(10^{21} \mathrm{~cm}^{-2}\right)$ & $F_{2.12 \mu \mathrm{m}}^{2}$ & $N_{\mathrm{Hx}}^{2}$ & $N_{\mathrm{H} \mathrm{NLR}}^{2}$ \\
$(1)$ & $(2)$ & $(3)$ & $(4)$ & $(5)$ & $(6)$ & $(7)$ \\
\hline Mrk 1210 & 42.36 & 0.83 & $0.286^{3}$ & $2.00 \pm 0.12$ & 0.32 & 0.58 \\
NGC 3227 & 42.00 & 0.50 & 0.036 & $17.7 \pm 1.00$ & 1.29 & 0.68 \\
NGC 4051 & 41.20 & 6.30 & $0.156^{3}$ & $5.81 \pm 0.37$ & 0.16 & 1.51 \\
NGC 4151 & 43.00 & 2.26 & 0.380 & $14.7 \pm 0.55$ & 1.45 & 24.6 \\
Mrk 766 & 43.00 & 1.28 & $0.260^{3}$ & $2.36 \pm 0.24$ & 0.67 & 1.68 \\
NGC 5548 & 43.76 & 4.57 & 0.021 & $0.80 \pm 0.11$ & 0.74 & 283.0 \\
NGC 5728 & 41.34 & $\ldots$ & $0.391^{3}$ & $6.28 \pm 0.12$ & $\ldots$ & 0.19 \\
\hline
\end{tabular}

Notes. Column 1 - object name; Col. 2 - Logarithmic 2-10 keV luminosity; Col. 3 - hydrogen column density determined from X-ray observations; Col. 4 - hydrogen column density for the NLR determined either from radio $21 \mathrm{~cm}$ observations or the $E(B-V)$; Col. $5-$ observed $\mathrm{H}_{2} 2.12 \mu$ m flux; Cols. 6-7 - predicted $\mathrm{H}_{2} 2.12 \mu$ m flux using column density from X-ray and radio, respectively.

${ }^{1}$ ASCA 2-10 keV luminosities were taken from George et al. (1998) for NGC 3227, NGC 4051, NGC 4151, Mrk 776 and NGC 5548 and from Awaki et al. (2000) for Mrk 1210.

${ }^{2}$ In units of $10^{-15} \mathrm{erg} \mathrm{s}^{-1} \mathrm{~cm}^{-2}$.

${ }^{3}$ Determined from the relation $N_{\mathrm{HI}}=5.2 \times 10^{21} E(B-V)$. See text for further details.

and Knop et al. (2001) showed that hard X-rays can do this for a sample of Seyfert 2 galaxies. Additional evidence for the role of X-rays can be drawn from the good correlation between $\mathrm{H}_{2} 2.121 \mu \mathrm{m} / \mathrm{Br} \gamma$ and [O I] $6300 \AA / \mathrm{H} \alpha$ and between [Fe II] $1.257 \mu \mathrm{m} / \mathrm{Pa} \beta$ and [O I] $6300 \AA \AA \mathrm{H} \alpha$ reported by Larkin et al. (1998). They argue that hard X-ray heating from a powerlaw source is a plausible mechanism to explain LINERS with low values of the $\left[\mathrm{Fe}{ }_{\mathrm{II}}\right] / \mathrm{Pa} \beta$ and $\mathrm{H}_{2} 2.121 \mu \mathrm{m} / \mathrm{Br} \gamma$ ratio, and which they suggest, are low-luminosity Seyfert galaxies. This same picture can be extended to the Seyfert galaxies of our sample to support the slim correlation reported here.

\section{2. $X$-rays as a source of [Fe II] and $\mathrm{H}_{2}$ lines}

In order to test if the observed [Fe $\mathrm{II}]$ and $\mathrm{H}_{2}$ emission can be attributed to X-ray heating, we have calculated the emergent $\mathrm{H}_{2} 2.121 \mu \mathrm{m}$ and [Fe II] $1.644 \mu \mathrm{m}$ fluxes using the models of X-ray excitation of Maloney et al. (1996). These models predict the resultant infrared emission (lines and continuum) of a neutral gas cloud of electron density $n_{\mathrm{e}}$ exposed to a source of hard X-rays of brightness $L_{\mathrm{x}}$ located at a distance $d$ from the cloud. The main parameter controlling the physical state of the $\mathrm{X}$-ray-illuminated gas is the effective ionization parameter, $\zeta_{\text {eff }}$, which is the attenuated X-ray flux to density ratio, determined from the expression

$\zeta_{\text {eff }} \simeq 100 \frac{L_{44}}{n_{5} d_{\mathrm{pc}}^{2} N_{22}^{0.9}}$,

where $L_{44}$ is the hard X-ray luminosity, $L_{\mathrm{x}}$, in units of $10^{44} \mathrm{erg} \mathrm{s}^{-1}, n_{5}$ is the gas (total hydrogen) density in units of $10^{5} \mathrm{~cm}^{-3}, d_{\mathrm{pc}}$ is the distance, in parsecs, to the AGN from the emitting gas, and $N_{22}$ is the attenuating column density between the AGN and the emitting gas, in units of $10^{22} \mathrm{~cm}^{-2}$. Once $\zeta_{\text {eff }}$ is computed, the predicted $\mathrm{H}_{2}$ and [Fe II] emergent intensities can be obtained with the help of Figs. 6a and $6 \mathrm{~b}$ of Maloney et al. (1996) for a gas density of $10^{5} \mathrm{~cm}^{-3}$ and $10^{3} \mathrm{~cm}^{-3}$, respectively.

The input parameters employed in our modeling are listed in Cols. 2-4 of Table 9. Two values of the hydrogen column density, $N_{\mathrm{H}}$, are listed and were used in the modeling: one determined directly from X-rays (Col. 3) and one from $21 \mathrm{~cm}$ radio observation (Col. 4). If this last value was not available, we used instead the $N_{\mathrm{H}}$ derived from $E(B-V)$ according to the expression $N_{\mathrm{H}}=5.2 \times 10^{21} E(B-V) \mathrm{cm}^{-2}$ (Shull \& van Steenberg 1985), which assumes Galactic dust/gas ratio. The distance $d$ between the $\mathrm{X}$-ray source and $\mathrm{H}$ i cloud was assumed to be equal to the radius of the integrated region (see Col. 10 of Table 1). This value represents the maximum distance at which the line could be emitted.

As a first test, we determined the predicted $\mathrm{H}_{2} 2.121 \mu \mathrm{m}$ intensity for a gas density $n_{\mathrm{e}}=10^{5} \mathrm{~cm}^{-3}$. The results appears in Cols. 6 and 7 of Table 9, for $N_{\mathrm{H}}$ derived from X-rays or either radio observations or $E(B-V)$, respectively. Compared to the observations, $\mathrm{H}_{2}$ intensities calculated using $N_{\mathrm{H}}$ obtained from X-ray data offer a poorer fit. This can be understood if we take into account that the hydrogen column density derived from hard $\mathrm{X}$-rays maps material obscuring the innermost part of active galaxies, while the $\mathrm{H}_{2}$ and [Fe II] emission is expected to arise farther out in the NLR. For this reason, we opted to use the $N_{\mathrm{H}}$ values determined either from $21 \mathrm{~cm}$ measurements or $E(B-V)$. With these constraints, we calculated the emergent $\left[\mathrm{Fe}\right.$ II] and $\mathrm{H}_{2}$ intensities and the results are listed in Table 10.

The results presented in Table 10 suggest that X-ray heating is responsible for only a fraction of the [Fe II $]$ and $\mathrm{H}_{2}$ emission, except in NGC 5548 where it can fully drive it. In four out of seven objects, the predicted [Fe II] flux is negligible while in NGC 4151 and NGC 3227, X-rays may contribute up to $15 \%$ of the measured [Fe II]. Regarding $\mathrm{H}_{2}$, X-rays contribute to the observed emission but only in NGC 4151 
Table 10. X-ray model parameters and predicted line fluxes for [Fe II] $1.64 \mu \mathrm{m}$ and $\mathrm{H}_{2} 2.12 \mu \mathrm{m}$.

\begin{tabular}{|c|c|c|c|c|c|c|}
\hline \multirow[b]{2}{*}{ Galaxy } & \multirow{2}{*}{$\begin{array}{c}n_{\mathrm{e}} \\
\left(\mathrm{cm}^{-3}\right)\end{array}$} & \multirow{2}{*}{$\begin{array}{c}N_{\mathrm{H}} \\
\left(10^{21} \mathrm{~cm}^{-2}\right)\end{array}$} & \multicolumn{2}{|c|}{ [Fe $\left.{ }_{\text {II }}\right] 1.64 \mu \mathrm{m}^{1}$} & \multicolumn{2}{|c|}{$\mathrm{H}_{2} 2.12 \mu \mathrm{m}^{1}$} \\
\hline & & & Obs. & Predicted & Obs. & Predicted \\
\hline Mrk 1210 & $10^{5}$ & 0.286 & $17.1 \pm 0.64$ & 0.0 & $2.00 \pm 0.12$ & 0.58 \\
\hline NGC 3227 & $10^{3}$ & 0.036 & $41.0 \pm 3.60$ & 7.08 & $17.7 \pm 1.00$ & 33.9 \\
\hline NGC 4051 & $10^{5}$ & 0.156 & $6.42 \pm 0.97$ & 0.0 & $5.81 \pm 0.37$ & 1.51 \\
\hline NGC 4151 & $10^{3}$ & 0.380 & $56.2 \pm 1.96$ & 10.7 & $14.7 \pm 0.55$ & 24.6 \\
\hline Mrk 766 & $10^{5}$ & 0.260 & $8.20 \pm 0.40$ & 0.0 & $2.36 \pm 0.24$ & 1.68 \\
\hline NGC 5548 & $10^{3}$ & 0.021 & $1.30 \pm 0.14$ & 15.8 & $0.80 \pm 0.11$ & 10.96 \\
\hline NGC 5728 & $10^{5}$ & 0.391 & $4.96 \pm 0.91$ & 0.0 & $6.28 \pm 0.12$ & 0.19 \\
\hline
\end{tabular}

${ }^{1}$ In units of $10^{-15} \mathrm{erg} \mathrm{s}^{-1} \mathrm{~cm}^{-2}$.

NGC 5548, and NGC 3227 can it entirely explain the excitation of the molecular gas. Note that for the Seyfert 2 galaxies Mrk 1210 and NGC 5728, an additional source of $\mathrm{H}_{2}$ would be necessary because X-rays contribute to only a small fraction of the measured flux.

The above discrepancy can be alleviated if the emitting clouds were effectively located closer to the X-ray source than the conservative distance we adopted in the models (equals to the beam size of our observations). In Mrk 1210, for instance, neutral clouds with $n_{\mathrm{e}}=10^{3} \mathrm{~cm}^{-3}$ at $\sim 80$ pc can generate enough $\mathrm{H}_{2}$ and [Fe II] to account for the observations. A similar result is obtained for NGC 5728. This possibility would obviate the need for additional excitation mechanisms and readily explain the correlation observed in Fig. 6. In order to confirm or discard this scenario, data taken at a superior resolution to map the inner molecular gas and constrain its density and distance from the central source are necessary. For the time being, we are only in a position to state that overall, the role of X-ray heating in exciting $\mathrm{H}_{2}$ and [ $\mathrm{Fe}$ II] lines in AGN is confirmed but the strength at which it operates strongly varies from object to object.

Morphological effects, more visible in Seyfert 2 than in Seyfert 1 galaxies, can be behind the strong departure from the correlation of Fig. 6 presented by some objects of the sample. In effect, most of the galaxies with large $\mathrm{H}_{2} 2.121 \mu \mathrm{m} / \mathrm{Br} \gamma$ or $\left[\mathrm{Fe}_{\mathrm{II}}\right] 1.257 \mu \mathrm{m} / \mathrm{Pa} \beta$ ratios share similar morphological aspects. For example, the presence of ionization cones and, most importantly, dust lanes, favour the production of $\mathrm{H}_{2}$ over [Fe II]. This could be the case in NGC 4945 and NGC 5728, where such features are clearly detected (Reunanen et al. 2002; Wilson et al. 1993). In these objects, $\mathrm{H}_{2} 2.121 \mu \mathrm{m} / \mathrm{Br} \gamma>2$. Jets and outflows, on the other hand, tend to favour [Fe II] emission over $\mathrm{H}_{2}$. This is the case in the galaxies NGC 2110 (which shows one of the $[\mathrm{Fe} I \mathrm{II}] / \mathrm{Pa} \beta$ ratios ever measured in an AGN, Knop et al. 2001), NGC 5128 (Centaurus A), and Mrk 3. In this case, $[\mathrm{Fe}$ II $] 1.257 \mu \mathrm{m} / \mathrm{Pa} \beta>2$. Whether these features are in fact related to the enhancement of any of the two emissions cannot be deduced from our data. Higher spatial resolution is needed to address this question.

Finally, it is interesting to note that X-rays models can explain $[\mathrm{Fe} \mathrm{II}] / \mathrm{Br} \gamma \sim 20$, as found by Alonso-Herrero et al. (1997), but it would need quite a high X-ray flux or clouds located too close to the central source. None of the objects studied (except probably NGC 5548), has the right combination of X-ray emission, hydrogen column density, and distance from the X-ray source to the emitting clouds, to produce ratios as high as 20 (using Maloney's models). Since these variables were constrained from observations, the results reflect the conditions for the galaxies of our sample.

\subsection{The [Fe II] lines as useful indicators of reddening for the NLR}

The simultaneous measurements of $[\mathrm{Fe} \mathrm{II}] 1.257 \mu \mathrm{m}$ and $1.644 \mu \mathrm{m}$ allow us to test, for the first time on such a big sample of AGN, how theory compares to observation. The ratio [Fe II] $1.257 \mu \mathrm{m} / 1.644 \mu \mathrm{m}$ is fixed by atomic physics to be 1.34 (Bautista \& Pradhan 1998) since both lines originate from the same upper level (i.e. ${ }^{4} \mathrm{D}_{7 / 2}-{ }^{6} \mathrm{D}_{9 / 2} /{ }^{4} \mathrm{D}_{7 / 2}-{ }^{4} \mathrm{~F}_{9 / 2}$ ). It means that this ratio is not only a good check on theoretical predictions but also a reliable indicator of the extinction towards the NLR because it is independent of temperature and density. Last column of Table 4 lists the [Fe II] $1.257 \mu \mathrm{m} / 1.644 \mu \mathrm{m}$ ratio measured in each galaxy. All but Mrk 279, NGC 5548 and Mrk 493, tend to have ratios $\sim 30 \%$ lower than the theoretical prediction. In fact, the mean [Fe II] $1.257 \mu \mathrm{m} / 1.644 \mu \mathrm{m}$ of the sample equals 0.98 . The uncertainties in the observed line ratios are $10 \%-20 \%$.

The above result can be interpreted in two ways. Either the the bulk of [ $\left.\mathrm{Fe}_{\mathrm{II}}\right]$ arises in a dusty region, or the $A$-values for the transitions involved need a fine tuning. In the first case, it implies that the extinction affecting the gas emitting the [Fe II] lines is large and surprisingly homogeneous. Assuming an intrinsic $1.257 \mu \mathrm{m} / 1.644 \mu \mathrm{m}$ ratio of 1.34 , the observed ratios agree with $A_{\mathrm{v}} \sim 3.7$ (adopting the Cardelli et al. 1989 extinction law and foreground screen). This last value would be even higher in Mrk 1239 and NGC 4051 but overall, rather similar in most AGN. Note that the extinction towards the NLR determined by other authors for some of the objects of our sample is significantly lower: $A_{\mathrm{v}} \sim 0.6$ for NGC 3227 (Crenshaw et al. 2001), $\sim 0.43$ for Ark 564 (Crenshaw et al. 2002) and $\sim 1.27$ for Mrk 766 (González Delgado \& Pérez 1996).

In the second case, our data can be useful to set important constraints to a more precise determination of the $A$-values 
for [Fe $\mathrm{II}$ ] forbidden lines. Bautista \& Pradhan (1998) had already noted the difficulty associated with the determination of accurate forbidden transition probabilities, especially for Fe II, owing to the large number of algebraic terms involved. Unfortunately, there are very few works published in the literature with a systematic determination of the ratio $I(1.257 \mu \mathrm{m} / 1.644 \mu \mathrm{m})$ on a similar sample. However, our results tend to agree to that of Sugai et al. (1999), who report a ratio of 0.80 for ARP 299. The extinction $A_{\mathrm{v}}$, determined by Heisler \& De Roberties (1999) from $I(1.257 \mu \mathrm{m} / 1.644 \mu \mathrm{m})$ on a small sample of $\mathrm{H}$ II and Seyfert 2 galaxies, also tends to be significantly higher that that derived from optical data.

In short, our observations imply that the extinction measured from the $\left[\mathrm{Fe}_{\mathrm{II}}\right]$ lines is large compared to that determined from optical indicators. This can be due to a genuine effect and it is expected because the NIR probes a larger optical depth than the optical. An alternative is to consider that the intrinsic $1.257 \mu \mathrm{m} / 1.644 \mu \mathrm{m}$ ratio is $\sim 30 \%$ smaller than the actual theoretical value. This is certainly an issue that deserves further attention and need to be explored using a larger sample of objects.

\section{Final remarks}

In order to discuss the origin of the molecular hydrogen and [Fe II] emission lines, near infrared observations have been carried out for a sample of 13 NLS1, 6 Seyfert 1, 2 Seyfert 2 and 1 starburst galaxies. In the following, our main conclusions are briefly reviewed.

The kinematical link between the molecular hydrogen and the NLR gas was analysed on the basis of the line widths. For all galaxies in the sample, $\mathrm{H}_{2} 2.121 \mu \mathrm{m}$ was in the limit of the spectral resolution $\left(360 \mathrm{~km} \mathrm{~s}^{-1}\right)$ and narrower than the NLR lines for the cases in which the latter lines were resolved. [Fe II] was resolved for $50 \%$ of the sample. In NGC 3227, NGC 4151 and PG 1612+261 it was broader than [S vI $1.963 \mu \mathrm{m}$. It is then unlikely that $\mathrm{H}_{2}$ and [Fe II] come from the same volume of gas.

The detection of $\mathrm{H}_{2}(1,0) \mathrm{S}(1) 2.121 \mu \mathrm{m}$ in 20 out of the 22 objects of our sample reveals, for the first time, strong evidence that molecular gas within $500 \mathrm{pc}$ from the centre is common in AGN and not restricted to SB/AGN composite galaxies. $\mathrm{H}_{2}$ lines from vibrational transitions were detected but are not as bright as in planetary nebulae, providing the first indication that UV fluorescence is not the main mechanism that excites them. This result is strengthened by the analysis of the line ratios that discriminates between shock-dominated and fluorescence production. For 4 objects (NGC 3227, NGC 4051, NGC 4151 and Mrk 766) the excitation mechanism is clearly thermal. For the remaining AGN, a mixing with a non-thermal process cannot be discarded, although the results point to a dominant thermal mechanism. This result is confirmed by the similarity between the vibrational and rotational temperatures of the $\mathrm{H}_{2}$. However, a unique source of the thermal component could not be established. The relative contribution to this component coming from shocks originating in $\mathrm{SN}$ explosions or in radio jets, and from $\mathrm{X}$-rays heating may vary from one object to the other.
The origin of the [Fe II] lines is also controversial. [Fe II] $1.257 \mu \mathrm{m}$ was detected in all galaxies of our sample, suggesting a link to the AGN itself. The [Fe II] $1.257 \mu \mathrm{m} / \mathrm{Pa} \beta$ ratio is used to study the origin of the [Fe II] emission. It reveals that stellar and non-stellar processes contributes to the [Fe II] spectrum with a predominance of the latter in most objects. The [Fe II] $1.257 \mu \mathrm{m} / \mathrm{Pa} \beta$ ratio values are within the interval usually observed in other AGN. Mrk 766 is particular in this respect, however. Being a NLS1, the observed [Fe II] is compatible with a starburst origin.

A correlation between $\mathrm{H}_{2} \quad 2.121 \mu \mathrm{m} / \mathrm{Br} \gamma$ and [Fe II] $1.257 \mu \mathrm{m} / \mathrm{Pa} \beta$ was found but it breaks down for the galaxies where either one of the ratios is larger than 2, predominantly Seyfert 2 s. Starburst galaxies are preferentially located in the region with $\left[\mathrm{Fe}{ }_{\mathrm{II}}\right] / \mathrm{Pa} \beta$ and $\mathrm{H}_{2} / \mathrm{Br} \gamma<0.4$ while LINERS are characterized by $[\mathrm{Fe}$ II $] / \mathrm{Pa} \beta$ and $\mathrm{H}_{2} / \mathrm{Br} \gamma>2$. We confirm the usefulness of this plot as a diagnostic tool at separating emitting line objects by activity level and set constraints to the locus of points shown by AGN.

Line intensities predicted by X-ray heating models are able to explain the $\mathrm{H}_{2}$ and part of the [Fe II] emission in Seyfert 1 but fail at reproducing these characteristics in Seyfert 2. The results are improved if the emitting clouds are located closer to the central source, $\sim 80 \mathrm{pc}$ or less. This would explain the correlation between $\mathrm{H}_{2} 2.121 \mu \mathrm{m} / \mathrm{Br} \gamma$ and [Fe II] $1.257 \mu \mathrm{m} / \mathrm{Pa} \beta$ discussed above. However, the presence of significant starburst activity in some of the objects makes it plausible that more than one exciting mechanism is at work. In Seyfert 2, X-rays appears to have a lesser importance and the $\mathrm{H}_{2}$ emission is probably enhanced by contributions from circumnuclear starburst and structures such as ionization cones and dust lanes. Nonetheless, the role of these structures is not confirmed by the present data.

Finally, our data allowed us to study, for the first time, the [Fe $\left.{ }_{\text {II }}\right] 1.257 \mu \mathrm{m} / 1.644 \mu \mathrm{m}$ ratio in Seyfert 1 galaxies. We found that most objects have values $30 \%$ lower than the intrinsic value predicted by theory. This implies either that the extinction towards the [Fe II] emitting clouds is very similar in most objects and significantly larger than that derived from optical observations, or there is an overestimation of the $A$-values for the Fe II transitions. We discarded a systematic error on the measurements because the uncertainties associated with the observed ratio are between $10 \%-20 \%$.

Acknowledgements. This paper is partially supported by the Brazilian funding agencies FAPESP (00/06695-0) and CNPq(304077/77-1 and 309054/03-6) to A.R.A. and S.M.V. and the U.S. National Science Foundation (A.K.P.). The authors are grateful for the comments and suggestions of the anonymous referee, which helped to improve this paper. This research has made use of the NASA/IPAC Extragalactic Database (NED) which is operated by the Jet Propulsion Laboratory, California Institute of Technology, under contract with the National Aeronautics and Space Administration.

\section{References}

Alonso-Herrero, A., Rieke, M. J., Rieke, G. H., \& Ruiz, M. 1997, ApJ, 482,747

Awaki, H., Ueno, S., Taniguchi, Y., \& Weaver, K. A. 2000, ApJ, 542, 175 
Bautista, M. A., \& Pradhan, A. K. 1998, ApJ, 492, 650

Black, J. H., \& van Dishoeck, E. F. 1987, ApJ, 322, 412

Blietz, M., Cameron, M., Drapatz, S., et al. 1994, ApJ, 421, 92

Braatz, J. A., Wilson, A. S., \& Henkel, C. 1994, ApJ, 437, L99

Cardelli, J. A., Clayton, G. C., \& Mathis, J. S. 1989, ApJ, 345, 245

Chrysostomou, A., Brand, P. W. J. L., Burton, M. G., \& Moorhouse, A. 1993, MNRAS, 265, 329

Crenshaw, D. M., Kraemer, S. B., Bruhweiler, F. C., \& Ruiz, J. R. 2001, ApJ, 555, 663

Crenshaw, D. M., Kraemer, S. B., Turner, T. J., et al. 2002, ApJ, 566, 187

Colina, L. 1993, ApJ, 411, 565

Cushing, M. C., Vacca, W. D., \& Rayner, J. T. 2003, PASP, submitted

De Robertis, M. M., \& Osterbrock, D. E. 1984, ApJ, 286, 171

Evans, I. N. 1988, ApJS, 67, 373

Forbes, D. A., \& Ward, M. J. 1993, ApJ, 416, 150

George, I. M., Turner, T. J., Netzer, H., et al. 1998, ApJS, 114, 73

González Delgado, R. M., \& Pérez, E. 1996, MNRAS, 278, 737

Goodrich, R. W., Veilleux, S., \& Hill, G. J. 1994, ApJ, 422, 521

Harrison, A., Puxley, P., Russel, A., \& Brand, P. 1998, MNRAS, 297, 624

Heisler, C. A., \& De Robertis, M. M. 1999, AJ, 118, 2038

Hollenbach, D., \& McKee, C. F. 1989, ApJ, 342, 306

Imanishi, M., Terada, H., Goto, M., \& Maihara, T. 1998, PASJ, 50, 399

Kawara, K., Nishida, M., \& Taniguchi, Y. 1988, ApJ, 328, L41

Knop, R. A., Armus, L., Larkin, J. E., et al. 1996, AJ, 112, 81

Knop, R. A., Armus, L., Matthews, K., Murphy, T. W., \& Soifer, B. T. 2001, AJ, 122, 764

Larkin, J. E., Armus, L., Knop, R. A., Soifer, B. T., \& Matthews, K. 1998, ApJS, 114, 59

Maloney, P. R., Hollenbach, D. J., \& Tielens, A. G. G. M. 1996, ApJ, 466, 561

Martini, P., Sellgren, K., \& Hora, J. 1997, ApJ, 484, 296

Mizutani, K., Suto, H., \& Maihara, T. 1994, ApJ, 421, 475

Moore, D., Cohen, R. D., \& Marcy, G. W. 1996, ApJ, 470, 280

Moorwood, A. F. M., \& Oliva, E. 1988, A\&A, 203, 278

Mouri, H., Kawara, K., Taniguchi, Y., \& Nishida, M. 1990, ApJ, 356, L39
Mouri, H., Kawara, K., \& Taniguchi, Y. 1993, ApJ, 406, 52

Mouri, H. 1994, ApJ, 427, 777

Mouri, H., Kawara, K., \& Taniguchi, Y. 2000, ApJ, 528, 186

Penston, M. V., Fosbury, R. A. E., Boksenberg, A., Ward, M. J., \& Wilson, A. S. 1984, MNRAS, 208, 347

Pogge, R. W., \& Owen, J. M. 1993, OSU Internal Report 93-01

Quillen, A. C., Alonso-Herrero, A., Rieke, M. J., et al. 1999, ApJ, 527, 696

Raluy, F., Planesas, P., \& Colina, L. 1998, A\&A, 335, 113

Rayner, J. T., Toomey, D. W., Onaka, P. M., et al. 2003, PASP, 155, 362

Reunanen, J., Kotilainen, J. K., \& Prieto, M. A. 2002, MNRAS, 331, 154

Rigolopoulou, D., Kunze, D., Lutz, D., Genzel, R., \& Moorwood, A. F. M. 2002, A\&A, 389, 374

Rodríguez-Ardila, A., Viegas, S., Pastoriza, M. G., \& Prato, L. 2002, ApJ, 519, 214

Rodríguez-Ardila, A., \& Viegas, S. 2003, MNRAS, 340, L33

Rudy, R. J., Lynch, D. K., Mazuk, S., Puetter, R. C., \& Dearborn, D. S. P. 2001, AJ, 121, 362

Schulz, H., \& Henkel, C. 2003, A\&A, 400, 41

Simpson, C., Forbes, D. A., Baker, A. C., \& Ward, M. J. 1996, MNRAS, 283, 777

Shull, J. A., \& Van Steenberg, M. E. 1985, ApJ, 294, 599

Shupe, D. L., Larkin, J. E., Knop, R. A., et al. 1998, ApJ, 498, 267

Sternberg, A., \& Dalgarno, A. 1989, ApJ, 338, 197

Storchi-Bergmann, T., Fernandes, R. C., \& Schmitt, H. R. 1998, ApJ, 501, 94

Sturm, E., Alexander, T., Lutz, D., et al. 1999, ApJ, 512, 197

Sugai, H., Davies, R. I., Malkan, M. A., et al. 1999, ApJ, 527, 778

Tuner, J. E. H., Allington-Smith, J., Chapman, S., et al. 2002, MNRAS, 331, 284

Vacca, W. D., Cushing, M. C., \& Rayner, J. T. 2003, PASP, 155, 389

Veilleux, S. 1991, ApJS, 75, 383

Veilleux, S., Goodrich, R. W., \& Hill, G. J. 1997, ApJ, 477, 631

Veilleux, S., Sanders, D. B., \& Kim, D.-C. 1997, ApJ, 484, 92

Wilson, A. S., Braatz, J. A., Heckman, T. M., Krolik, J. H., \& Miley, G. K. 1993, ApJ, 419, L61 